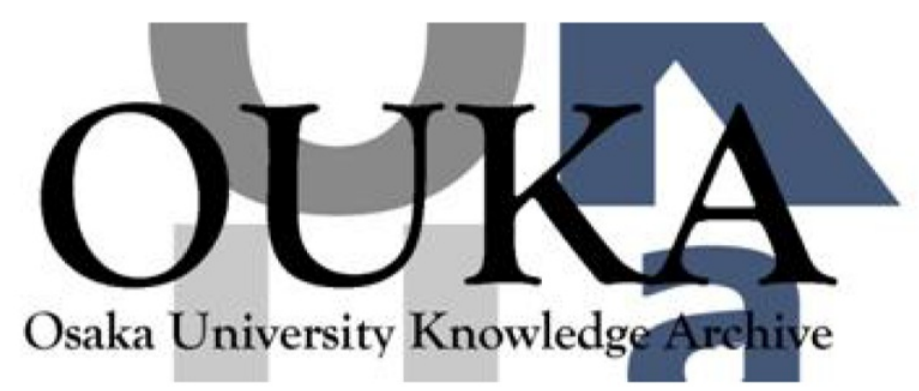

\begin{tabular}{|c|l|}
\hline Title & Higgs inflation from standard model criticality \\
\hline Author(s) & Hamada, Yuta; Kawai, Hikaru; Oda, Kinya et al. \\
\hline Citation & Physical Review D. 91(5) p. 053008 \\
\hline Issue Date & $2015-03-26$ \\
\hline oaire:version & VoR \\
\hline URL & https://hdl. handle. net/11094/78743 \\
\hline rights & ○ 2015 American Physical Society \\
\hline Note & \\
\hline
\end{tabular}

Osaka University Knowledge Archive : OUKA

https://ir. Library. osaka-u. ac. jp/

Osaka University 


\title{
Higgs inflation from standard model criticality
}

\author{
Yuta Hamada, ${ }^{1, *}$ Hikaru Kawai, ${ }^{1, \dagger}$ Kin-ya Oda, ${ }^{2, \ddagger}$ and Seong Chan Park ${ }^{3, \S}$ \\ ${ }^{1}$ Department of Physics, Kyoto University, Kyoto 606-8502, Japan \\ ${ }^{2}$ Department of Physics, Osaka University, Osaka 560-0043, Japan \\ ${ }^{3}$ Department of Physics, Sungkyunkwan University, Suwon 440-746, Korea \\ and Korea Institute for Advanced Study, Seoul 130-722, Korea
}

(Received 24 August 2014; published 26 March 2015)

\begin{abstract}
The observed Higgs mass $M_{H}=125.9 \pm 0.4 \mathrm{GeV}$ leads to the criticality of the standard model, that is, the Higgs potential becomes flat around the scale $10^{17-18} \mathrm{GeV}$ for the top mass $171.3 \mathrm{GeV}$. Earlier we proposed a Higgs inflation scenario in which this criticality plays a crucial role. In this paper, we investigate the detailed cosmological predictions of this scenario in light of the latest Planck and BICEP2 results. We also consider the Higgs portal scalar dark matter model, and compute the Higgs one-loop effective potential with the two-loop renormalization group improvement. We find a constraint on the coupling between the Higgs boson and dark matter which depends on the inflationary parameters.
\end{abstract}

DOI: 10.1103/PhysRevD.91.053008

PACS numbers: $98.80 . \mathrm{Cq}, 14.80 . \mathrm{Bn}$

\section{INTRODUCTION}

The observed value of the Higgs mass [1]

$$
M_{H}=125.9 \pm 0.4 \mathrm{GeV}
$$

indicates that the standard model (SM) Higgs potential becomes small and flat at the scale around $10^{17-18} \mathrm{GeV}$ for the top mass $171.3 \mathrm{GeV}$; see e.g. [4-16] for the latest analyses. ${ }^{2}$ This fact suggests [34] that the Higgs field beyond the ultraviolet (UV) cutoff of the SM, at the criticality [35], may play the role of the slowly rolling inflaton in the early Universe; see Ref. [36] for the original proposal to use the Higgs field for the cosmological inflation and also Refs. [37-40] for the idea to use the false vacuum of the SM at criticality. Especially, under the presence of the large nonminimal coupling $\xi \sim 10^{4}$ between the Higgs field and the Ricci curvature, there arises a plateau in the SM effective potential above the field value $\varphi \sim M_{P} / \sqrt{\xi}$, and enough of a number of $e$-foldings is achieved without introducing any other field beyond the SM [36,41-46]. In Ref. [47], it has been shown by numerical analysis that smaller values of $\xi \sim 400$ and 90

\footnotetext{
*hamada@gauge.scphys.kyoto-u.ac.jp

†hkawai@gauge.scphys.kyoto-u.ac.jp

odakin@phys.sci.osaka-u.ac.jp

§.park@skku.edu

${ }^{1}$ The latest values of the Higgs mass are $125.03_{-0.27}^{+0.26}$ (stat) ${ }_{-0.15}^{+0.13}$ (syst) $\mathrm{GeV}$ (CMS) [2] and $125.36 \pm$ 0.37 (stat) \pm 0.18 (syst) GeV (ATLAS)[3], which are consistent with each other and also with the particle date group (PDG) value we are using here.

${ }^{2}$ It is an intriguing fact that the bare Higgs mass also becomes small at the same scale [9,17-19]; see also Refs. [20-24]. The running Higgs mass after the subtraction of the quadratic divergence is considered e.g. in Ref. [25]; see also Refs. [26-33].
}

are possible in the prescriptions I and II, respectively; see Sec. III for what prescription means.

In Ref. [48], we proposed to push the idea of Ref. [34] to use the criticality of the SM for the Higgs inflation scenario in order to accommodate a lower value of $\xi=7-100$, as well as a wider range of the tensor-to-scalar ratio $r \lesssim 0.2$; see also Refs. $[49,50] .{ }^{3}$ Similar attempts have been done in some extensions of the SM [48,51-55]. There have also been different directions of the extension of the Higgs inflation involving higher dimensional operators [56-63]. See also Refs. [64-92].

In this paper, we give detailed analyses of the Higgs inflation scenario proposed in Ref. [48] that utilizes the saddle point, at which both the first and second derivatives of the potential become very small. The scale dependence of the effective quartic coupling $\lambda_{\text {eff }}$ is very important to determine the effective potential, whose behavior around the saddle point is characterized by the minimum value $\lambda_{\min }$ of the effective coupling $\lambda_{\text {eff }}$, the corresponding scale $\mu_{\min }$, and the second derivative $\beta_{2}$ of $\lambda_{\text {eff }}$ around $\mu_{\min }$, in addition to $\xi$. We examine the predictions of this model on spectral index $n_{s}$, tensor-to-scalar ratio $r$, and the running of spectral index $\mathrm{d} n_{s} / \mathrm{d} \ln k$.

We also estimate how small the higher dimensional Planck-suppressed operators must be in order to maintain the observed values of the cosmic microwave background (CMB). For that purpose, we pick up the six-dimensional operator $\varphi^{6} / M_{P}^{2}$ in the Jordan-frame potential as a concrete example, and compute the CMB spectral indices.

We also evaluate the relation between the high-scale parameters $\mu_{\min }, \beta_{2}$ and the low energy parameters in the SM, as well as in the Higgs portal scalar dark matter (DM)

\footnotetext{
${ }^{3}$ See Sec. V A 3 for an explanation for the apparent discrepancy between the results from Refs. [48,50] and those from Ref. [47,49].
} 
model, using one-loop effective potential and the two-loop renormalization group equation (RGE).

This paper is organized as follows. In Sec. II, we review the criticality, namely the flatness and smallness, of the SM Higgs potential around the scale $10^{17-18} \mathrm{GeV}$. In Sec. III, we review the Higgs inflation scenario in a wider perspective. In Sec. IV, we investigate the predictions of this model in detail. In Sec. V, we consider the extension with the Higgs portal scalar DM. We summarize our results in the last section.

\section{STANDARD MODEL HIGGS POTENTIAL}

In the SM on the flat spacetime background, the oneloop effective potential calculated in the $\overline{\mathrm{MS}}$ scheme in the Landau gauge is

$$
V=V_{\text {tree }}+\Delta V_{\text {1-loop }}
$$

with

$$
\begin{aligned}
& V_{\text {tree }}=e^{4 \Gamma(\varphi)} \frac{\lambda(\mu)}{4} \varphi^{4}, \\
& \Delta V_{1 \text {-loop }}=e^{4 \Gamma(\varphi)}\left\{-\frac{3 m_{t}(\varphi)^{4}}{16 \pi^{2}}\left(\ln \frac{m_{t}(\varphi)^{2}}{\mu^{2}}-\frac{3}{2}+2 \Gamma(\varphi)\right)\right. \\
& \left.+\frac{6 m_{W}(\varphi)^{4}}{64 \pi^{2}}\left(\ln \frac{m_{W}(\varphi)^{2}}{\mu^{2}}-\frac{5}{6}+2 \Gamma(\varphi)\right)+\frac{3 m_{Z}(\varphi)^{4}}{64 \pi^{2}}\left(\ln \frac{m_{Z}(\varphi)^{2}}{\mu^{2}}-\frac{5}{6}+2 \Gamma(\varphi)\right)\right\}, \\
& \Gamma(\varphi)=\int_{M_{t}}^{\varphi} \gamma d \ln \mu, \\
& \gamma=\frac{1}{(4 \pi)^{2}}\left(\frac{9}{4} g_{2}^{2}+\frac{3}{4} g_{Y}^{2}-3 y_{t}^{2}\right),
\end{aligned}
$$

where $m_{W}(\varphi)=g_{2} \varphi / 2, m_{Z}(\varphi)=\sqrt{g_{Y}^{2}+g_{2}^{2}} \varphi / 2$, and $m_{t}(\varphi)=y_{t} \varphi / \sqrt{2}$. We have neglected the effects from the loops of the Higgs and would-be Nambu-Goldstone bosons since we are interested in the scale where $\lambda$ becomes small. We also neglect the quadratic term; the bare Higgs mass is canceled by the loop effect at low energies; see e.g. Appendix B in Ref. [34].

We define the effective quartic coupling as [6]

$$
V(\varphi)=\frac{\lambda_{\mathrm{eff}}(\varphi, \mu)}{4} \varphi^{4} .
$$

At the one-loop level,

$$
\begin{aligned}
\lambda_{\mathrm{eff}}(\varphi, \mu)= & e^{4 \Gamma(\varphi)} \lambda(\mu)+e^{4 \Gamma(\varphi)} \frac{1}{16 \pi^{2}}\left[-3 y_{t}^{4}\left(\ln \frac{y_{t}^{2} \varphi^{2}}{2 \mu^{2}}-\frac{3}{2}+2 \Gamma(\varphi)\right)+\frac{3 g_{2}^{4}}{8}\left(\ln \frac{g_{2}^{2} \varphi^{2}}{4 \mu^{2}}-\frac{5}{6}+2 \Gamma(\varphi)\right)\right. \\
& \left.+\frac{3\left(g_{Y}^{2}+g_{2}^{2}\right)^{2}}{16}\left(\ln \frac{\left(g_{Y}^{2}+g_{2}^{2}\right) \varphi^{2}}{4 \mu^{2}}-\frac{5}{6}+2 \Gamma(\varphi)\right)\right],
\end{aligned}
$$

where we have made the scale dependence explicit in the right-hand side while omitting it in $y_{t}, g_{2}$, and $g_{Y}$, which correspond to the two-loop corrections.

In the $\mathrm{SM}$ on the flat spacetime background, $\Delta V_{1 \text {-loop }}$ is minimized by

$$
\begin{aligned}
\ln \frac{\varphi^{2}}{\mu^{2}} & =\frac{C_{t}^{2}\left(-\ln C_{t}+\frac{3}{2}-2 \Gamma\right)-2 C_{W}^{2}\left(-\ln C_{W}+\frac{5}{6}-2 \Gamma\right)-C_{Z}^{2}\left(-\ln C_{Z}+\frac{5}{6}-2 \Gamma\right)}{C_{t}^{2}-2 C_{W}^{2}-C_{Z}^{2}} \\
& =\frac{\left(-\ln C_{t}+\frac{3}{2}-2 \Gamma\right)-2 \frac{C_{W}^{2}}{C_{t}^{2}}\left(-\ln C_{W}+\frac{5}{6}-2 \Gamma\right)-\frac{C_{Z}^{2}}{C_{t}^{2}}\left(-\ln C_{Z}+\frac{5}{6}-2 \Gamma\right)}{1-2 \frac{C_{W}^{2}}{C_{t}^{2}}-\frac{C_{Z}^{2}}{C_{t}^{2}}},
\end{aligned}
$$

where $C_{W}=g_{2}^{2} / 4, C_{Z}=\left(g_{Y}^{2}+g_{2}^{2}\right) / 4$, and $C_{t}=y_{t}^{2} / 2$. Around $\mu_{\min } \sim 10^{17-18} \mathrm{GeV}$, Eq. (9) leads to $\mu \simeq 0.23 \varphi$. However, because the difference of the numerical values of the one-loop effective potential for $\mu=\varphi$ and $\mu=0.23 \varphi$ is negligibly small, we use $\mu=\varphi$ hereafter in this section. 
Then we obtain

$$
V(\varphi)=\frac{\lambda_{\mathrm{eff}}(\mu=\varphi)}{4} \varphi^{4}
$$

where $\lambda_{\text {eff }}(\mu)$ is written by

$\lambda_{\mathrm{eff}}(\mu)=e^{4 \Gamma} \lambda(\mu)+e^{4 \Gamma} \frac{1}{16 \pi^{2}}\left[-3 y_{t}^{4}\left(\ln \frac{y_{t}^{2}}{2}-\frac{3}{2}+2 \Gamma\right)+\frac{3 g_{2}^{4}}{8}\left(\ln \frac{g_{2}^{2}}{4}-\frac{5}{6}+2 \Gamma\right)+\frac{3\left(g_{Y}^{2}+g_{2}^{2}\right)^{2}}{16}\left(\ln \frac{\left(g_{Y}^{2}+g_{2}^{2}\right)}{4}-\frac{5}{6}+2 \Gamma\right)\right]$,

at the one-loop level.

The effective coupling $\lambda_{\text {eff }}$ is quartically sensitive to $y_{t}$ and thus the top quark mass, $M_{t}$, which is scheme dependently defined. The actual value of $M_{t}$ is known with large uncertainties at the level of $\mathrm{GeV}$ scales depending on the measurements:

$$
\begin{gathered}
M_{t}^{\text {pole }}= \begin{cases}171.2 \pm 2.4 \mathrm{GeV}, & \text { MITP [99], } \\
176.7_{-3.4}^{+4.0} \mathrm{GeV}, & \text { PDG [136], }\end{cases} \\
M_{t}^{\text {Pythia }}= \begin{cases}173.21 \pm 0.51 \pm 0.71 \mathrm{GeV}, & \text { direct measurment, PDG [136] } \\
174.98 \pm 0.76 \mathrm{GeV}, & \text { D0[137], } \\
174.34 \pm 0.64 \mathrm{GeV}, & \text { D0 }+ \text { CDF [138], } \\
173.34 \pm 0.76 \mathrm{GeV}, & \text { ATLAS [139], } \\
172.38 \pm 0.10 \pm 0.65 \mathrm{GeV}, & \text { CMS [140]. }\end{cases}
\end{gathered}
$$

One should note that the "directly measured value" in Eq. (13) obtained by Tevatron (D0 and CDF) and by LHC (ATLAS and CMS) is indeed a parameter in Monte Carlo simulation code [7,93], the so-called Pythia mass [94], whose physical relation to the pole and $\overline{\mathrm{MS}}$ masses is not well established. In discussing the Higgs inflation near criticality, however, the only important fact is that the critical value for the pole mass $M_{t} \simeq 171.3 \mathrm{GeV}$, shown just below, is perfectly consistent with both the mainz institute for theoretical physics (MITP) and PDG within $2 \sigma$ confidence level. Below, we take the MITP value as a benchmark.

In Fig. 1, we can see that $\lambda_{\text {eff }}$ has the minimum around $10^{17-18} \mathrm{GeV}$. Interestingly, if $M_{t} \simeq 171.3 \mathrm{GeV}$, the minimum value of $\lambda_{\text {eff }}$ becomes zero around the scale $10^{17-18} \mathrm{GeV}$, and the Higgs potential has a plateau around $10^{17-18} \mathrm{GeV}$ as shown in Fig. $2{ }^{4}$

Let us expand the effective potential of the Higgs field $V_{\text {eff }}(\varphi)$ on the flat spacetime background around its minimum in terms of $\ln \varphi$ :

\footnotetext{
${ }^{4}$ It has been known that such a position of plateau is unphysical and can vary by an order of magnitude depending on the gauge choice [95]. The gauge dependence of the effective potential can be absorbed by a field redefinition [96]. The eventual field equation for $\varphi$ should not depend on such a choice, but the field value here necessarily contains this amount of uncertainty. See also Refs. [97,98] for a further account on the gauge (in) dependence.
}

$$
\begin{aligned}
V(\varphi) & =\frac{\lambda_{\mathrm{eff}}(\mu=\varphi)}{4} \varphi^{4}, \\
\lambda_{\mathrm{eff}}(\mu) & =\lambda_{\min }+\sum_{n=2}^{\infty} \frac{\beta_{n}}{\left(16 \pi^{2}\right)^{n}}\left(\ln \frac{\mu}{\mu_{\mathrm{min}}}\right)^{2},
\end{aligned}
$$

where the overall factor $\varphi^{4}$ is put to make the expansion well behaved. In the potential analysis around the minimum, we can safely neglect the higher order terms with

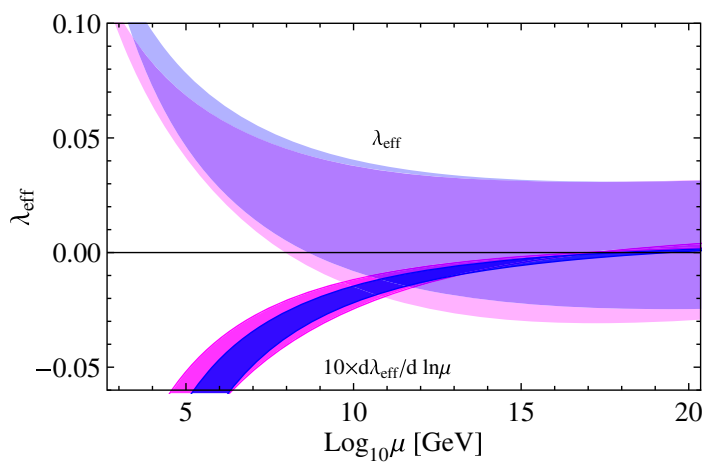

FIG. 1 (color online). The light red (lower) and blue (upper) bands are two-loop RGE running of $\lambda(\mu)$ and $\lambda_{\text {eff }}(\mu)$ (11), respectively. The dark red (upper) and blue (lower) bands are the beta function times ten $10 \times \mathrm{d} \lambda_{\text {eff }} / \mathrm{d} \ln \mu$ evaluated at the tree and one-loop levels, respectively. We take $M_{H}=125.9 \mathrm{GeV}$ and $\alpha_{s}=0.1185$. The band corresponds to $95 \%$ C.L. deviation of $M_{t}$ [99]; see Eq. (12). 

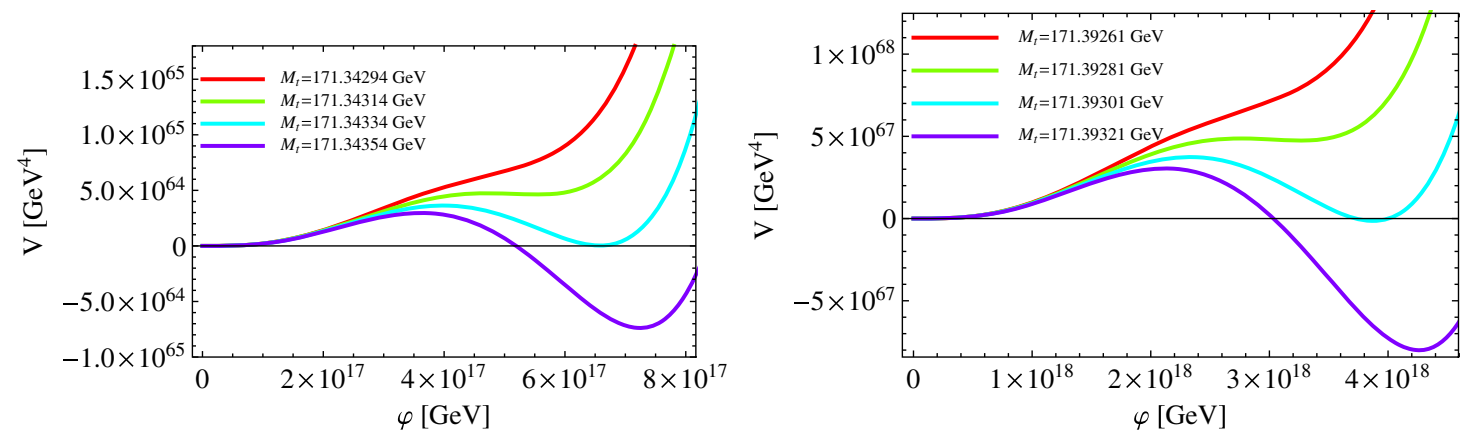

FIG. 2 (color online). Left: The tree-level Higgs potential (3) as a function of Higgs field $\varphi$. Right: The one-loop Higgs potential (3)-(4). We take $M_{H}=125.9 \mathrm{GeV}$ and $\alpha_{s}=0.1185$.
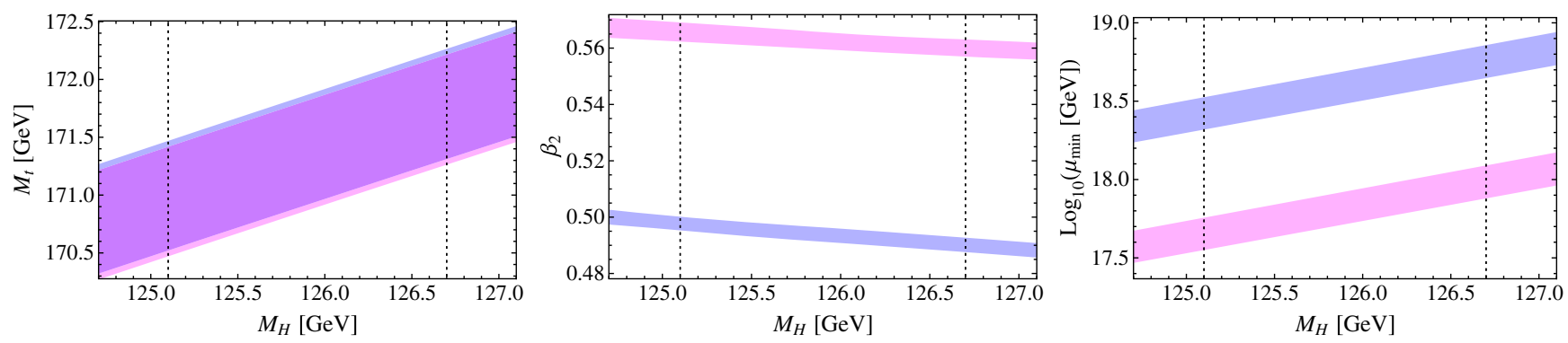

FIG. 3 (color online). $\quad M_{t}$ (left), $\beta_{2}$ (center), and $\mu_{\min }$ (right) that realize the condition $\lambda_{\min }=\lambda_{c}$ are plotted as functions of $M_{H}$. We have imposed the condition $\lambda_{\min }=\lambda_{c}$ using the tree-level potential (3) and the one-loop one, (3)-(4), for the red and blue bands, respectively. (The one-loop blue band is the upper one for left and right, whereas it is the lower for center.) The width of the bands corresponds to the 95\% C.L. of $\alpha_{s}\left(M_{Z}\right)$. Dotted lines show the current 95\% C.L. for $M_{H}$; see Eq. (1).

$n \geq 3$, and omit them hereafter. By tuning the top mass for a given Higgs mass, we can obtain arbitrarily small $\lambda_{\min }$. This fact is crucial for our inflation scenario.

We note that for the potential to be monotonically increasing [48], $\lambda_{\min }$ must be larger than a critical value $\lambda_{c}$ :

$$
\lambda_{\min } \geq \lambda_{c}:=\frac{\beta_{2}}{\left(64 \pi^{2}\right)^{2}}
$$

When $\lambda_{\min }$ saturates this inequality,

$$
\lambda_{\min }=\lambda_{c}
$$

there appears a true saddle point of the potential $V_{\varphi}=V_{\varphi \varphi}=0$. We see in Sec. VA that in prescription $\mathrm{I}$, this value $\lambda_{c}$ also gives the true saddle point of the modified potential: $U_{\varphi}=U_{\varphi \varphi}=0 .{ }^{5}$

In the left, center, and right of Fig. 3 , we plot $M_{t}, \beta_{2}$, and $\mu_{\min }$, respectively, with the critical value of $\lambda_{\min }$ given in

\footnotetext{
${ }^{5}$ The numerical difference between the results from the condition $\lambda_{\min }=0$ and from Eq. (16) is much smaller than the deviation coming from the $\alpha_{s}\left(M_{Z}\right)$ error. We have imposed $\lambda_{\min }=0$ within a precision of $10^{-5}$ in the actual numerical computation in writing Fig. 3 . Note that $\lambda_{c}=2.5 \times 10^{-6} \beta_{2}$.
}

Eq. (16). The band corresponds to the 95\% C.L. for the strong coupling constant measured at $\mu=M_{Z}$, where

$$
\alpha_{s}\left(M_{Z}\right)=0.1185 \pm 0.0006
$$

at the $1 \sigma$ level [1]. We see that $\beta_{2}$ does not depend much on $M_{H}$. In the following figures except Fig. 12, we take a reference value $\beta_{2}=0$. . $^{6} \mu_{\min }$ changes by an order of magnitude when one includes the one-loop corrections to the effective potential as shown in the right of Fig. 3. The two-loop corrections are negligible compared with the oneloop corrections; see e.g. Ref. [6]. In Fig. 3, we see that $\beta_{2}$ and $\mu_{\min }$ differ between tree and one-loop levels, but note that $M_{t}$ is almost identical at both levels.

\section{INFLATION MODEL}

Let us consider the effective action of the SM-gravity system in the local potential approximation. As we are interested in the spatially constant field configuration and the case where the Hubble parameter is much smaller than the Planck scale, we restrict ourselves to the terms

\footnotetext{
${ }^{6}$ We have checked that the changes of spectral index, its running, its running of running, and tensor-to-scalar ratio are hardly seeable when we vary $\beta_{2}$.
} 
containing up to the second derivative of the fields. We can write down the effective action schematically as ${ }^{7}$

$$
\begin{aligned}
S= & \int \mathrm{d}^{4} x \sqrt{-g}\left[\frac{M_{P}^{2}}{2} A(\varphi) \mathcal{R}-\frac{1}{2} B(\varphi) g^{\mu \nu}\left(\partial_{\mu} \varphi \partial_{\nu} \varphi+A_{\mu} A_{\nu} \varphi^{2}\right)-V(\varphi)-C(\varphi) \bar{\psi} \gamma^{\mu} D_{\mu} \psi\right. \\
& \left.-\frac{y}{\sqrt{2}} D(\varphi)(\varphi \bar{\psi} \psi+\text { H.c. })-\frac{E(\varphi)}{4 g_{A}^{2}} F_{\mu \nu} F^{\mu \nu}\right],
\end{aligned}
$$

where $g_{A}$ and $y$ are gauge and Yukawa couplings, respectively, $M_{P}:=1 / \sqrt{8 \pi G}=2.4 \times 10^{18} \mathrm{GeV}$ is the reduced Planck scale, $\varphi$ is the physical (real) Higgs field, and

$$
A(\varphi)=1+a_{2} \frac{\varphi^{2}}{M_{P}^{2}}+a_{4} \frac{\varphi^{4}}{M_{P}^{4}}+\cdots, \quad B(\varphi)=1+b_{2} \frac{\varphi^{2}}{M_{P}^{2}}+b_{4} \frac{\varphi^{4}}{M_{P}^{4}}+\cdots, \quad \text { etc. },
$$

with $a_{2}, \ldots, b_{2}, \ldots$, etc. being dimensionless constants. Generically the potential $V$ also contains higher dimensional terms

$$
g_{\mu \nu}^{E}=A(\varphi) g_{\mu \nu},
$$

to get the action in the Einstein frame

$$
\begin{aligned}
S= & \int \mathrm{d}^{4} x \sqrt{-g_{E}}\left[\frac{M_{P}^{2}}{2} \mathcal{R}_{E}-\frac{1}{2}\left[\frac{B(\varphi)}{A(\varphi)}+\frac{3}{2} \frac{B(\varphi) A^{\prime}(\varphi)^{2}}{A(\varphi)^{2}}\right] g_{E}^{\mu \nu} \partial_{\mu} \varphi \partial_{\nu} \varphi-\frac{1}{2}\left[\frac{B(\varphi)}{A(\varphi)}\right] g_{E}^{\mu \nu} A_{\mu} A_{\nu} \varphi^{2}-\frac{V(\varphi)}{A(\varphi)^{2}}\right. \\
& \left.-\frac{C(\varphi)}{A(\varphi)^{3 / 2}} \bar{\psi} \gamma_{E}^{\mu} D_{\mu} \psi-\frac{y}{\sqrt{2}} \frac{D(\varphi)}{A(\varphi)^{2}}(\varphi \bar{\psi} \psi+\text { H.c. })-\frac{E(\varphi)}{4 g_{A}^{2}} F_{\mu \nu} F_{E}^{\mu \nu}\right] ;
\end{aligned}
$$

see e.g. Refs. $[100,101]$.

By the field redefinition,

$\frac{\mathrm{d} \chi}{\mathrm{d} \varphi}=\sqrt{\frac{B(\varphi)}{A(\varphi)}+\frac{3}{2} \frac{B(\varphi) A^{\prime}(\varphi)^{2}}{A(\varphi)^{2}}}, \quad \tilde{\psi}=\frac{C(\varphi)^{1 / 2}}{A(\varphi)^{3 / 4}} \psi$

we get the canonically normalized kinetic term for $\chi$ and

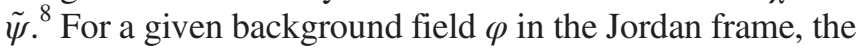
effective mass for the canonically normalized field $\tilde{\psi}$ is

$$
m_{\tilde{\psi}}=\frac{y \varphi}{\sqrt{2}} \frac{D(\varphi)}{\sqrt{A(\varphi)} C(\varphi)} .
$$

Similarly, the effective mass for a canonically normalized gauge field is

\footnotetext{
${ }^{7}$ In article [48], we used $h$ instead of $\varphi$.

${ }^{8}$ There appear extra derivative terms from the kinetic term of the fermion. We neglect such terms, since we are interested in the expression of the fermion mass for a constant background field $\varphi$ and for the Hubble parameter much smaller than the Planck scale.
}

$$
m_{\tilde{A}}=g_{A} \varphi \sqrt{\frac{B(\varphi)}{A(\varphi) E(\varphi)}} .
$$

For later convenience, we define the Einstein frame potential

$$
U(\varphi):=\frac{V(\varphi)}{A(\varphi)^{2}}
$$

In the original version of the Higgs inflation $[36,44,45]$, it is assumed that $\xi:=a_{2}$ happens to be large: $\xi \sim 10^{4}$, whereas the other couplings are not much larger than unity: $\xi \gg a_{2}, a_{4}, \ldots ; b_{2}, \ldots$, etc. In that limit, we can write

$$
\begin{aligned}
A(\varphi) & =1+\frac{\xi \varphi^{2}}{M_{P}^{2}}, \\
B(\varphi) & =C(\varphi)=D(\varphi)=E(\varphi)=1, \\
\lambda_{6} & =\lambda_{8}=\ldots=0 .
\end{aligned}
$$

As a side remark, we note that we can instead assume $b_{2} \sim 10^{5}$ while keeping all other coefficients, including $a_{2}$, 
not much larger than unity in order to realize another version of Higgs inflation [61]. It may be interesting to look for more possibilities of putting a large number in other places. In this paper, we restrict ourselves to a more conventional set of the nonminimal couplings (27), and later take into account the term $\lambda_{6} \varphi^{6}$ in the potential (20) as a next step.

For $\varphi \gg M_{P} / \sqrt{\xi}$, we have $\mathrm{d} \chi / \mathrm{d} \varphi \simeq \sqrt{6} M_{P} / \varphi$, which leads to $\varphi \simeq \frac{M_{P}}{\sqrt{\xi}} \exp \left(\chi / \sqrt{6} M_{P}\right)$, and the potential becomes [36]

$$
U(\chi)=\frac{V}{\left(1+e^{2 \chi / \sqrt{6} M_{P}}\right)^{2}} .
$$

The analysis of this model without taking into account the running of $\lambda$ gives the following predictions [36]:

$$
\begin{aligned}
n_{s} & =1-6 \epsilon_{V}+2 \eta_{V} \simeq 0.967, \\
r & =16 \epsilon_{V} \simeq 3 \times 10^{-3}, \\
\frac{\mathrm{d} n_{s}}{\mathrm{~d} \ln k} & =16 \epsilon_{V} \eta_{V}-24 \epsilon_{V}^{2}-2 \zeta_{V}^{2} \simeq-5.4 \times 10^{-4},
\end{aligned}
$$

where

$$
\begin{gathered}
\epsilon_{V}=\frac{M_{P}^{2}}{2}\left(\frac{\mathrm{d} U / \mathrm{d} \chi}{U}\right)^{2}, \\
\eta_{V}=M_{P}^{2} \frac{\mathrm{d}^{2} U / \mathrm{d} \chi^{2}}{U}, \\
\zeta_{V}^{2}=M_{P}^{4} \frac{\left(\mathrm{d}^{3} U / \mathrm{d} \chi^{3}\right)(\mathrm{d} U / \mathrm{d} \chi)}{U^{2}} .
\end{gathered}
$$

As is seen in Eq. (8), the loop corrections to the effective potential contain large logarithms. They can be written as $\ln (M(\varphi) / \mu)$, where $\mu$ is the renormalization scale and $M(\varphi)$ stands for the field-dependent mass of the particle running in the loop, namely the top quark and the gauge bosons. The problem is that there are two possibilities in defining the field-dependent mass [44]. In the so-called prescription I, we use the field-dependent mass in the Einstein frame, as in Eqs. (24)-(25), whereas in prescription II, we use the ones in the Jordan frame, namely $m_{\psi}=$ $y \varphi / \sqrt{2}$ and $m_{A}=g_{A} \varphi$. We leave the possibilities open for future research and present our results for both prescriptions.

As we have done below Eq. (9), in either prescription I or II, we can drop the gauge and Yukawa couplings in the field-dependent mass. For prescription I, assuming the minimal set of coefficients (27), we put

$$
\mu=\frac{\varphi}{\sqrt{1+\xi \varphi^{2} / M_{P}^{2}}}
$$

and for prescription II,

$$
\mu=\varphi
$$

Therefore, the effective potential is

$$
V=\frac{\lambda_{\mathrm{eff}}(\mu)}{4} \varphi^{4},
$$

with the scale (33) for prescription I and scale (34) for prescription II, where $\lambda_{\text {eff }}(\mu)$ in the SM is given by Eq. (11).

\section{COSMOLOGICAL CONSTRAINTS}

The overall normalization of the CMB fluctuation fixes [102]

$$
A_{s}=\frac{V}{24 \pi^{2} \epsilon_{V} M_{P}^{4}}=\left(2.196_{-0.058}^{+0.053}\right) \times 10^{-9},
$$

within $1 \sigma$ C.L. Current Planck + WMAP bounds on the spectral index, its running, its running of running, and the tensor-to-scalar ratio are [102]

$$
\begin{aligned}
& n_{s}=0.9514_{-0.0090}^{+0.0087}, \quad \frac{\mathrm{d} n_{s}}{\mathrm{~d} \ln k}=0.001_{-0.014}^{+0.016}, \quad \frac{\mathrm{d}^{2} n_{s}}{\mathrm{~d} \ln k^{2}}=0.020_{-0.015}^{+0.016}, \quad r=0 \text { (assumed), }
\end{aligned}
$$

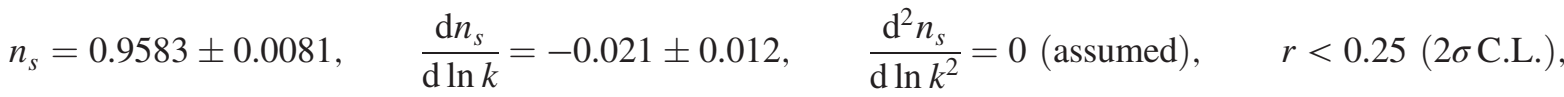

at the pivot scale $k_{*}=0.05 \mathrm{Mpc}^{-1}$, within $1 \sigma$ C.L. unless otherwise stated. The BICEP2 experiment has reported an observation of $r$ [103]:

$$
r=0.20_{-0.05}^{+0.07}
$$

within $1 \sigma$ C.L.
It has been pointed out that the BICEP2 result may become consistent with $r=0$ because the foreground effect can be sizable $[104,105]$. We also note that by including isocurvature perturbation, the $95 \%$ C.L. bound on $n_{s}$ is roughly loosened to [106]

$$
0.93 \lesssim n_{s} \lesssim 0.99
$$


and that by including sterile neutrinos, the allowed range is shifted to [107]

$$
0.95 \lesssim n_{s} \lesssim 1.02
$$

Given the above, we plot our results within wider ranges than those in Eqs. (37)-(38):

$$
\begin{gathered}
0.93 \leq n_{s} \leq 1.02, \\
-0.05 \leq \frac{\mathrm{d} n_{s}}{\mathrm{~d} \ln k} \leq 0.05, \\
0 \leq r \leq 0.3 .
\end{gathered}
$$

\section{HIGGS INFLATION FROM STANDARD MODEL CRITICALITY}

In this section, we start from the minimal set of coefficients (27), and later include the term $\lambda_{6} \varphi^{6}$.
We expand the effective potential of the Higgs field $V_{\text {eff }}$ on the flat spacetime background around its minimum as in Eq. (14):

$$
\begin{gathered}
V=\frac{\lambda_{\mathrm{eff}}(\mu)}{4} \varphi^{4}, \\
\lambda_{\mathrm{eff}}(\mu)=\lambda_{\min }+\sum_{n=2}^{\infty} \frac{\beta_{n}}{\left(16 \pi^{2}\right)^{n}}\left(\ln \frac{\mu}{\mu_{\mathrm{min}}}\right)^{2} .
\end{gathered}
$$

The choice of scale (33)-(34) corresponds to prescription I and II, respectively. As in Sec. II, we can safely neglect the higher order terms with $n \geq 3$, and we continue to omit them.

\section{A. Prescription I}

\section{Analysis in prescription I}

In prescription I, the Higgs potential is given by Eqs. (26) and (44) with the scale (33). Concretely,

$$
\begin{aligned}
U(\varphi) & =\frac{\varphi^{4}}{4\left(1+\xi \varphi^{2} / M_{P}^{2}\right)^{2}}\left\{\lambda_{\min }+\frac{\beta_{2}}{\left(16 \pi^{2}\right)^{2}}\left[\ln \left(\frac{1}{c} \sqrt{\frac{\xi \varphi^{2} / M_{P}^{2}}{1+\xi \varphi^{2} / M_{P}^{2}}}\right)\right]^{2}\right\} \\
U^{\prime}(\varphi) & =\frac{\varphi^{3} M_{P}^{6}}{\left(M_{P}^{2}+\xi \varphi^{2}\right)^{3}}\left\{\lambda_{\min }+\frac{\beta_{2}}{2\left(16 \pi^{2}\right)^{2}}\left[1+2 \ln \left(\frac{1}{c} \sqrt{\frac{\xi \varphi^{2}}{M_{P}^{2}+\xi \varphi^{2}}}\right)\right] \ln \left(\frac{1}{c} \sqrt{\frac{\xi \varphi^{2}}{M_{P}^{2}+\xi \varphi^{2}}}\right)\right\},
\end{aligned}
$$

where we define $c$ by

$$
\mu_{\min }=c \frac{M_{P}}{\sqrt{\xi}}
$$

Note that we have defined $\mu_{\min }$ to give the minimum of the effective coupling $\lambda_{\text {eff }}\left(\mu_{\min }\right)=\lambda_{\min }$ on the flat spacetime background Eq. (45). The stationary points $U^{\prime}\left(\varphi_{1}\right)=0$ are given by

$$
\varphi_{1}=\frac{c M_{P}}{\sqrt{\xi}} \frac{1}{\left(e^{\frac{1}{2}\left[1 \pm \sqrt{1-\frac{\lambda_{\min }}{\lambda_{c}}}\right.}-c^{2}\right)^{1 / 2}} .
$$

We can see the following:

(i) When $\lambda_{\min }>\lambda_{c}$, the potential is a monotonically increasing function of $\varphi$. This case corresponds to the red (upper) line in Fig. 4.

(ii) When $\lambda_{\min }=\lambda_{c}$ :

(a) For $c \geq e^{1 / 4}$, the potential is monotonically increasing.

(b) For $c<e^{1 / 4}$, the potential has a stationary point at

$$
\varphi_{c}=\frac{c M_{P}}{\sqrt{\xi}} \frac{1}{\left(\sqrt{e}-c^{2}\right)^{1 / 2}} .
$$

In this case, $\varphi_{c}$ becomes a saddle point: $U^{\prime}\left(\varphi_{c}\right)=U^{\prime \prime}\left(\varphi_{c}\right)=0$. This case corresponds to the green (center) line in Fig. 4.

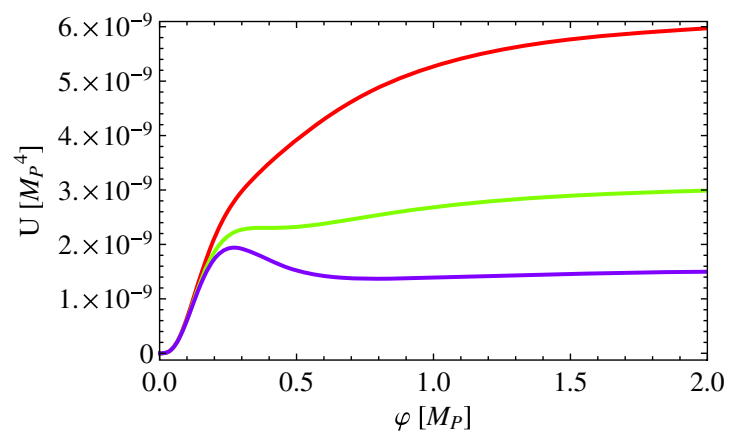

FIG. 4 (color online). SM Higgs potential in prescription I with $\xi=10$ and $c=1$, corresponding to $\mu_{\min }=7.6 \times 10^{17} \mathrm{GeV}$, and with $\beta_{2}=0.5$. The red (upper), green (center), and purple (lower) lines are drawn with $\lambda_{\min }=2 \lambda_{c}, \lambda_{c}$, and $\lambda_{c} / 2$, respectively. The values of $\lambda_{\min }=2 \lambda_{c}$ and $\lambda_{c} / 2$ are chosen just for illustration. Each line roughly corresponds to the one with the same color in Fig. 2. 

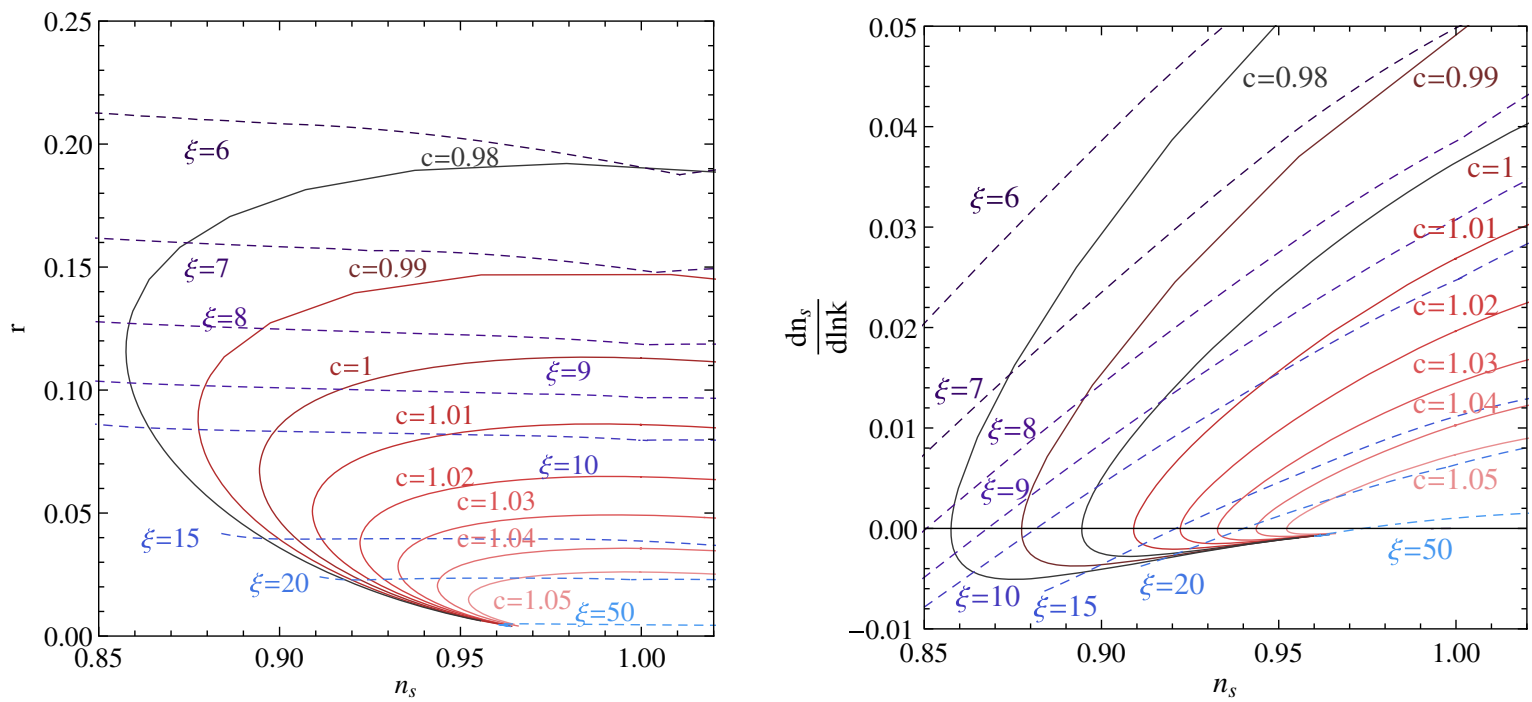

FIG. 5 (color online). Left: $r$ vs $n_{s}$. Right: $\mathrm{d} n_{s} / \mathrm{d} \ln k$ vs $n_{s}$. The solid and dashed contours are for fixed $c$ and $\xi$, respectively. The left end of each dashed line for $\xi=15,20$, and 50 corresponds to $c=0.94$. The lower end of each solid line corresponds to $\xi=50$.

(iii) When $\lambda_{\min }<\lambda_{c}$, we define $c_{ \pm}:=\exp \frac{1 \pm \sqrt{1-\frac{\lambda_{\min }}{\lambda_{c}}}}{4}$

(a) For $c \geq c_{+}$, the potential is monotonically increasing.

(b) For $c_{-}<c<c_{+}$, the potential has a stationary point given by the plus sign of Eq. (48).

(c) For $c \leq c_{-}$, the potential has two stationary points given by Eq. (48). This case corresponds to the (lower) line in Fig. 4.

In this paper, we pursue the possibility that $\lambda\left(\mu_{\min }\right) \simeq 0$ is realized by a principle beyond the ordinary local field theory, such as the multiple point criticality principle [35,108,109], classical conformality [26-31,110-112], asymptotic safety [113], the hidden duality and symmetry [114,115], and the maximum entropy principle [116-119].

In practice, this amounts to the following: $\mu_{\min }$ is fixed for a given set of $M_{H}$ and $\alpha_{s}\left(M_{Z}\right)$ in the SM. For a given $\mu_{\min }$, we require $\xi$ to sit in

$$
\xi=c^{2} \frac{M_{P}^{2}}{\mu_{\min }^{2}}<\sqrt{e} \frac{M_{P}^{2}}{\mu_{\min }^{2}}
$$

That is, we consider the case $c<e^{1 / 4}$. By tuning the top quark mass, we can always choose a $\lambda_{\min }$ that is very close but larger than $\lambda_{c}$ so that we realize $U^{\prime}(\varphi) \ll U(\varphi) / M_{P}$ and $U^{\prime \prime}(\varphi) \ll U(\varphi) / M_{P}^{2}$ around $\varphi \simeq \varphi_{c}{ }^{9}$. In Fig. 4, our choice is very close but slightly above the green (middle) line.

In extensions of the SM, $\mu_{\min }$ depends on newly added parameters too. Anyway, we require Eq. (50), and choose a

\footnotetext{
${ }^{9}$ More precisely, we need $U_{\chi} \ll U / M_{P}$ and $U_{\chi \chi} \ll U / M_{P}^{2}$, which are satisfied when $U_{\varphi} \ll U / M_{P}$ and $U_{\varphi \varphi} \ll U / M_{P}^{2}$ because we have $\mathrm{d} \varphi / \mathrm{d} \chi \sim 1 / \sqrt{6 \xi}$ during the inflation.
}

$\lambda_{\min }$ that is very close to $\lambda_{c}$, with $\lambda_{\min }>\lambda_{c}$, by the tuning of the top mass and possible other parameters.

We also need to consider the effect of the running of $\xi$ [120-123]. However, this effect is small. More concretely, if $\xi$ and $\varphi$ are sufficiently large, $\xi$ is given around $\mu=\mu_{\min }$ by [44]

$$
\begin{aligned}
\xi(\mu) & \simeq \xi_{0}\left\{1-\left(\frac{3}{2} g_{Y}^{2}+3 g_{2}^{2}-6 y_{t}^{2}\right) \frac{1}{16 \pi^{2}} \ln \frac{\mu}{\mu_{\min }}\right\} \\
& \simeq \xi_{0}\left\{1+0.001 \ln \frac{\mu}{\mu_{\min }}\right\} .
\end{aligned}
$$

We treat $\xi$ as a constant in this paper.

\section{Results in prescription I}

The Higgs potential is determined by three parameters, $\xi$, $c$, and $\lambda_{\min }$. Qualitatively, $\xi$ determines the total suppression of the potential above the scale $\phi \gtrsim M_{P} / \sqrt{\xi}, c$ determines the maximum value of $\epsilon_{V}$ above the almost-saddle point, and $\lambda_{\min }$ determines the number of $e$-foldings. We choose $\lambda_{\min }$ such that we can have sufficient $e$-folding $N=60$. For a fixed $A_{s}=2.2 \times 10^{-9}$, other cosmological parameters $n_{s}$, $r$, and $\mathrm{d} n_{s} / \mathrm{d} \ln k$ can be calculated as functions of $\xi$ and $c$.

We show the typical predictions of this model in Fig. 5. Each solid line corresponds to a constant $c$. Dashed lines correspond to the values of $\xi$ from 6 to 50 as indicated in the figure. In Fig. 5, we see that there is a minimum value of $\xi$ that can result in $r \lesssim 0.2$, namely $\xi_{\min } \sim 7$. The model can reproduce $r=O\left(10^{-3}\right) \sim 0.2$ and $n_{s}=0.9 \sim 1.0$. These predictions are consistent with the Planck or BICEP2 result $[102,103]$. However, the value of $\mathrm{d} n_{s} / \mathrm{d} \ln k$ is slightly large. The prediction is $\mathrm{d} n_{s} / \mathrm{d} \ln k=O(0.01)$ for $r \gtrsim 0.05$. In Sec. VA 4, we see that the introduction of small 

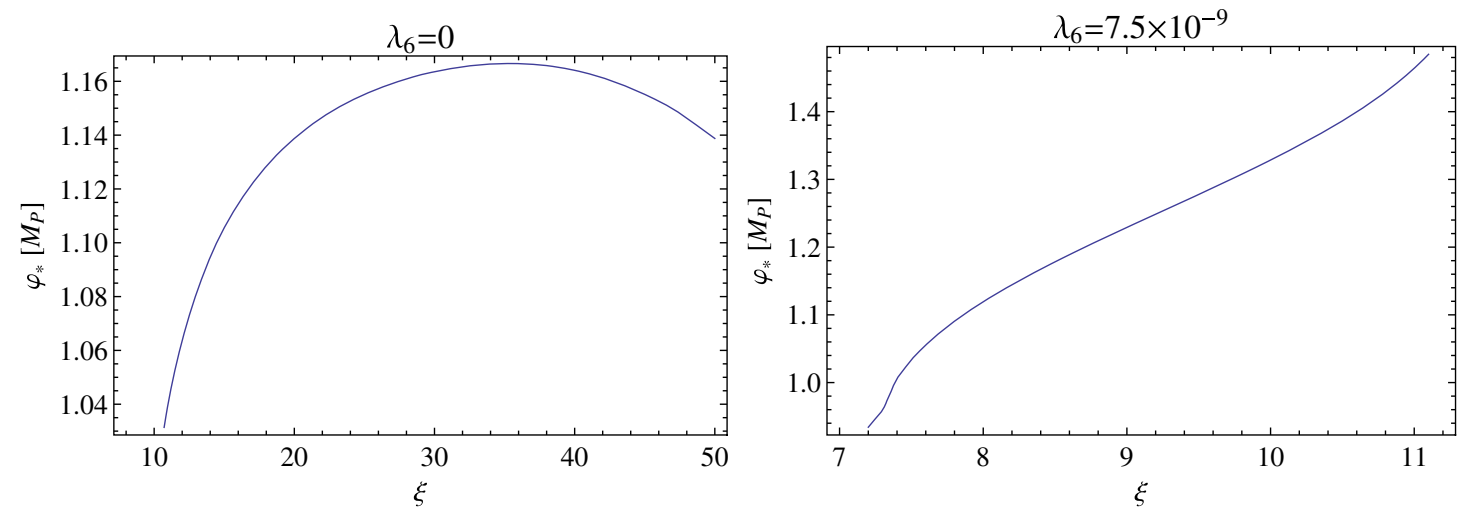

FIG. 6 (color online). Left and right: $\varphi_{*}$ as a function of $\xi$, with $\lambda_{6}=0$ and $7.5 \times 10^{-9}$, respectively. Other parameters are taken as $c=1$ and $\beta_{2}=0.5$.

coefficients of higher dimensional operators may ameliorate the situation.

Finally, we discuss the field value $\varphi_{*}$ that corresponds to the observed CMB fluctuation. The left panel of Fig. 6 shows $\varphi_{*}$ in the case of $c=1$ and $\beta_{2}=0.5 .{ }^{10}$ We see that $\varphi_{*}$ is around the Planck scale: $\varphi_{*} \sim M_{P}$.

\section{Constraint on $\mu_{\min }$}

The above analysis shows the existence of the lowest possible value of $\xi$, which is $\xi_{\min } \sim 7$. It is a necessary condition that $\mu_{\text {min }}$, which is obtained from the parameters at low energy, satisfies $\mu_{\text {min }} \lesssim M_{P} / \sqrt{\xi_{\text {min }}}$ for any successful Higgs inflation with $\xi>\xi_{\text {min }}$. However, as we have observed in Sec. II, SM one-loop effective potential takes its minimum above $M_{P} / \sqrt{\xi_{\text {min }}}$ although the tree-level potential can realize $\mu_{\min } \lesssim M_{P} / \sqrt{\xi_{\min }}$. It appears that it is difficult to do our Higgs inflation in SM.

However, taking into account the ambiguity coming from nonrenormalizable nonminimal coupling $\xi$, there still remains a possibility of realizing $\mu_{\min } \lesssim M_{P} / \sqrt{\xi_{\min }}[50]$. Around the scale $M_{P} / \xi$, we match $\lambda$ in the SM without $\xi$ and $\lambda_{\xi}$ in the SM with $\xi$ :

$$
\lambda=\lambda_{\xi}+(\text { threshold corrections }),
$$

where the threshold corrections generally contain power divergences and cannot be determined unless we specify a UV theory beyond the cutoff. One expects that the threshold corrections start from one-loop order. Because they are of the same order as the difference between the tree and one-loop effective potentials, it may result in $\mu_{\text {min }} \lesssim M_{P} / \sqrt{\xi_{\text {min }}}$. See also the similar discussion in footnote 4 regarding the gauge dependence.

In Sec. VI, we see that we can easily obtain $\mu_{\text {min }} \lesssim$ $M_{P} / \sqrt{\xi_{\text {min }}}$ in the Higgs portal scalar DM model without

\footnotetext{
${ }^{10}$ Precisely speaking, there are two $\varphi_{*}$ which satisfy Eq. (36) given $c, \xi$. We plot the one solution which gives more desirable predictions on cosmological parameters, namely, $n_{s} \lesssim 0.99$.
}

referring to such arguments. The argument of this section applies also to prescription II shown in Sec. V B.

\section{Estimation of the effects of higher dimensional operators in prescription I}

As we have seen in the previous sections, the extrapolation of the low energy data shows that the Higgs potential in the $\mathrm{SM}$ is flat around the string scale. This flatness can be broken if we introduce arbitrary strengths to the higher dimensional Planck-suppressed operators. In order to examine the effects of such operators on the cosmological data, we consider, for example, a small sixth order term in the Jordan frame

$$
\Delta V=\lambda_{6} \frac{\varphi^{6}}{M_{P}^{2}} .
$$

Here we do not claim that Eq. (53) gives the leading contribution among the higher dimensional operators, but simply estimate the ambiguity in the value of $\mathrm{d} n_{s} / \mathrm{d} \ln k$ discussed in the previous section. Of course, we can give any form to $\mathrm{d} n_{s} / \mathrm{d} \ln k$ as a function of $k$ if we introduce arbitrary strengths to the higher dimensional operators. As we will see below, the single term (53) allows the value of $\mathrm{d} n_{s} / \mathrm{d} \ln k$ at $k_{*}$ to reside in the favored region. However, this should not be taken as a prediction of the value of $\lambda_{6}$ but as an estimation for the allowed magnitude of the coefficients of the higher dimensional operators.

In the Einstein frame potential, the term (53) becomes

$$
\Delta U=\lambda_{6} \frac{\varphi^{6}}{\left(1+\xi \varphi^{2} / M_{P}^{2}\right)^{2}} .
$$

In Figs. 7-8, we plot the contours for fixed $\lambda_{6} \leq 10^{-8}$ with the solid lines, in the $r$ vs $n_{s}$ plane and the $\mathrm{d} n_{s} / \mathrm{d} \ln k$ vs $n_{s}$ one, respectively. We also plot the contours for fixed $\xi$ and $\lambda_{6}$ in the dashed and solid lines, respectively. We can realize the $r \simeq 0.1, n_{s} \simeq 0.96$, and $\mathrm{d} n_{s} / \mathrm{d} \ln k \simeq-0.01$ simultaneously. Finally, $\varphi_{*}$ has been plotted in the right panel of Fig. 6 with $\lambda_{6}=5 \times 10^{-9}, c=1$, and $\beta_{2}=0.5$. 

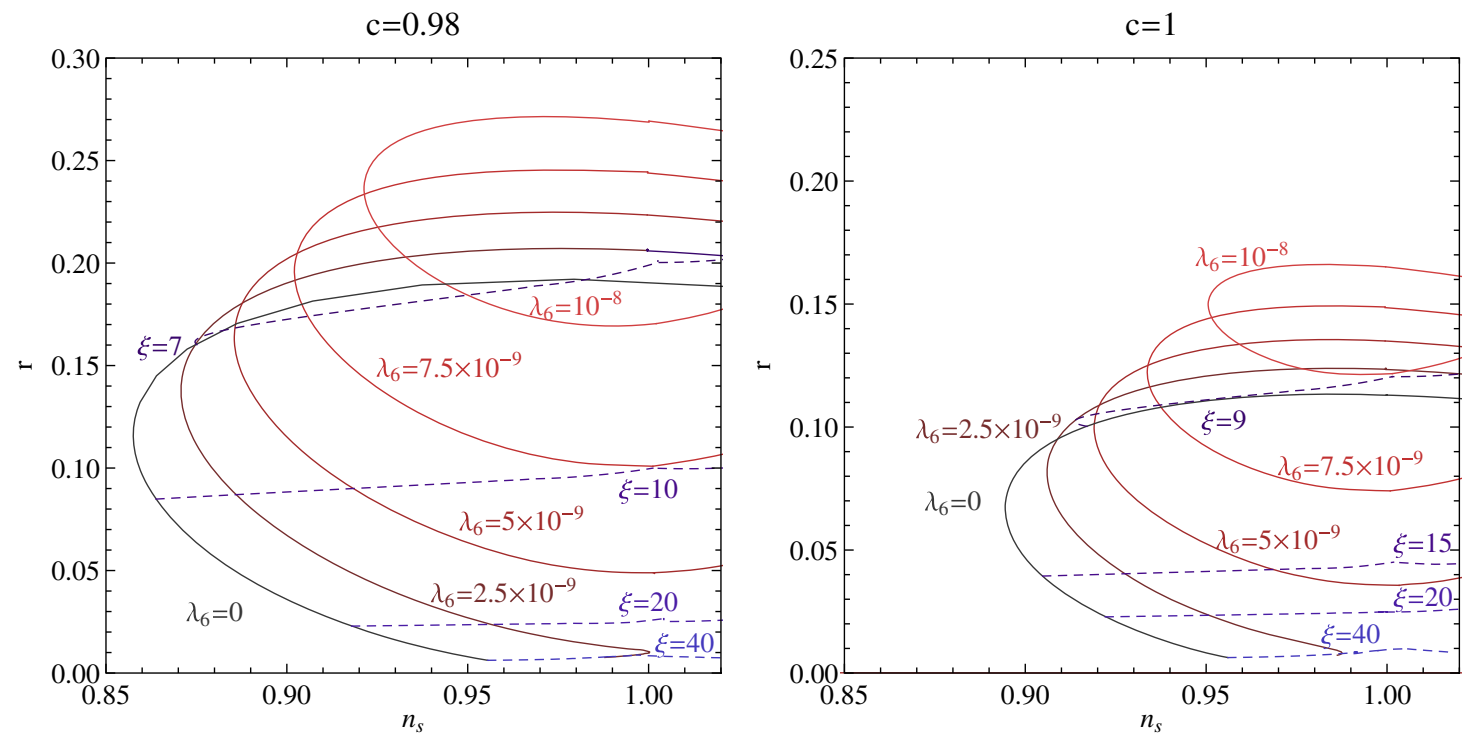

FIG. 7 (color online). $\quad r$ vs $n_{s}$, with $c=0.98$ (left) and 1 (right). Solid and dashed contours are for fixed $\lambda_{6}$ and $\xi$, respectively. We put $\beta_{2}=0.5$.
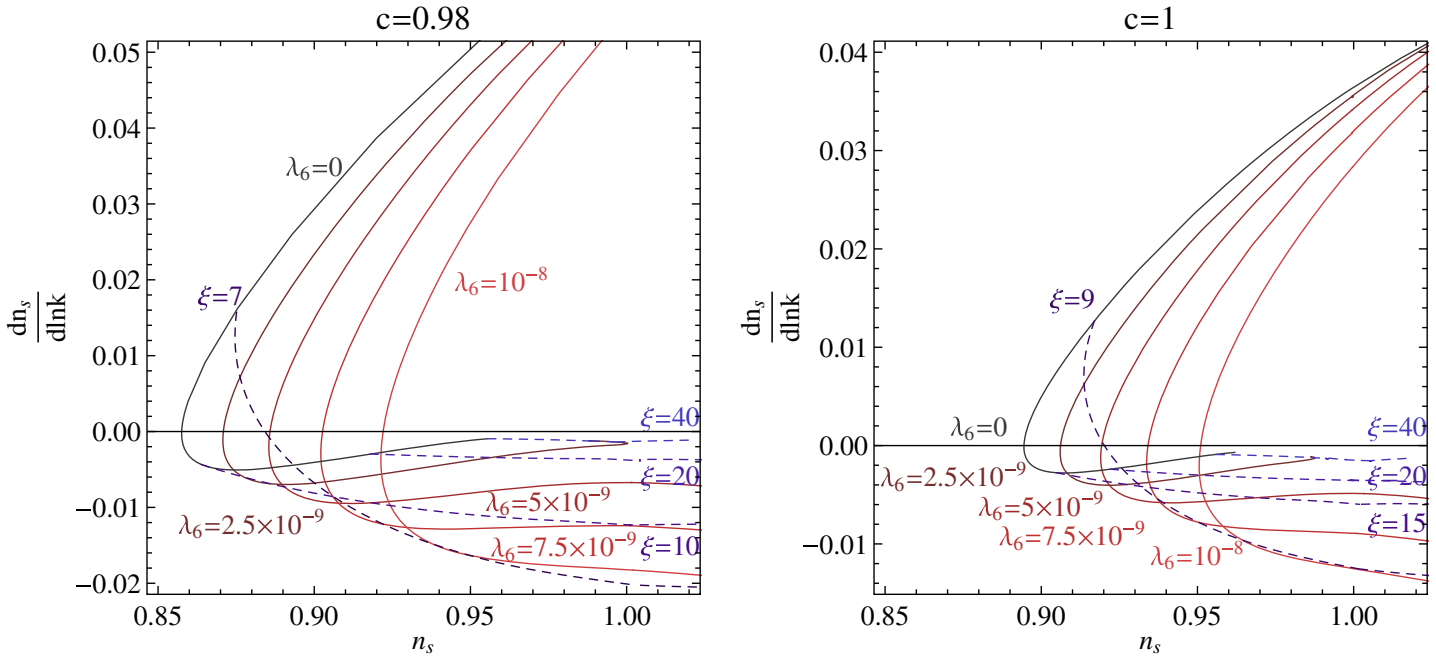

FIG. 8 (color online). $\quad \mathrm{d} n_{s} / \mathrm{d} \ln k$ vs $n_{s}$, with $c=0.98$ (left) and 1 (right). Solid and dashed contours are for fixed $\lambda_{6}$ and $\xi$, respectively. We put $\beta_{2}=0.5$.

The other higher dimensional operators should also have the coefficients $\lesssim \mathcal{O}\left(10^{-8}\right)$ in order to keep the flatness of the potential. Their smallness may be understood for example as a tiny explicit breaking of the asymptotic scale invariance in Jordan frame (the shift symmetry in Einstein frame) [124].

\section{B. Prescription II}

\section{Analysis in prescription II}

In prescription II, the Higgs potential is given by Eqs. (26) and (44) with $\mu=\varphi$,

$$
U(\varphi)=\frac{\lambda(\varphi)}{4} \frac{\varphi^{4}}{\left(1+\xi \varphi^{2} / M_{P}^{2}\right)^{2}},
$$

which gives

$$
U=\frac{X^{4}}{\left(1+X^{2}\right)^{2}} \frac{1}{4}\left(\lambda_{\min }+\frac{\beta_{2}}{\left(16 \pi^{2}\right)^{2}}\left(\ln \frac{X}{c}\right)^{2}\right)\left(\frac{M_{P}}{\sqrt{\xi}}\right)^{4},
$$




$$
U_{\varphi}=\frac{X^{3}}{\left(1+X^{2}\right)^{3}}\left\{\lambda_{\min }+\frac{\beta_{2}}{2\left(16 \pi^{2}\right)^{2}}\left(1+X^{2}\right) \ln \frac{X}{c}+\frac{\beta_{2}}{\left(16 \pi^{2}\right)^{2}}\left(\ln \frac{X}{c}\right)^{2}\right\}\left(\frac{M_{P}}{\sqrt{\xi}}\right)^{3},
$$

where $X=\frac{\varphi}{M_{P} / \sqrt{\xi}}$. Then the slow-roll parameter (30) becomes

$$
\epsilon_{V}=\frac{8 \xi}{X^{2}+(1+6 \xi) X^{4}} \frac{\left(\lambda_{\min }+\frac{\beta_{2}}{2\left(16 \pi^{2}\right)^{2}} \ln \frac{X}{c}\left(1+X^{2}+2 \ln \frac{X}{c}\right)\right)^{2}}{\left(\lambda_{\min }+\frac{\beta_{2}}{\left(16 \pi^{2}\right)^{2}}\left(\ln \frac{X}{c}\right)^{2}\right)^{2}} \text {. }
$$

For $\varphi \gg M_{P} / \sqrt{6} \xi$ and $\xi \gg 1 / 6$, we obtain

$$
\epsilon_{V} \simeq \frac{4}{3 X^{4}} \frac{\left(\lambda_{\min }+\frac{\beta_{2}}{2\left(16 \pi^{2}\right)^{2}} \ln \frac{X}{c}\left(1+X^{2}+2 \ln \frac{X}{c}\right)\right)^{2}}{\left(\lambda_{\min }+\frac{\beta_{2}}{\left(16 \pi^{2}\right)^{2}}\left(\ln \frac{X}{c}\right)^{2}\right)^{2}} .
$$

Similarly we have

$\eta_{V} \simeq \frac{4}{3 X^{4}}\left(1-X^{2}+\frac{\beta_{2}}{4\left(16 \pi^{2}\right)^{2}} \frac{\left(1+X^{2}\right)\left(1+X^{2}+6 \ln \frac{X}{c}\right)}{\lambda_{\min }+\frac{\beta_{2}}{\left(16 \pi^{2}\right)^{2}}\left(\ln \frac{X}{c}\right)^{2}}\right)$.

These expressions are in agreement with those in the original Higgs inflation [36] if we take $\beta_{2}=0$ and $X \gg 1$.

The $e$-folding $N$ is written by

$$
N=\int_{X_{\text {end }}}^{X_{*}} \frac{\mathrm{d} \chi}{M_{P}} \frac{1}{\sqrt{2 \epsilon_{V}}}=\int \frac{\mathrm{d} \chi}{\mathrm{d} X} \frac{\mathrm{d} X}{\sqrt{2 \epsilon_{V}}} \frac{1}{M_{P}}
$$

where

$$
\frac{\mathrm{d} \chi}{\mathrm{d} X}=\frac{\sqrt{1+(1+6 \xi) X^{2}}}{1+X^{2}} \frac{M_{P}}{\sqrt{\xi}} \simeq \frac{\sqrt{6 X}}{1+X^{2}} M_{P} .
$$

In the last step, we have used the same limit as above: $X \gg 1 / \sqrt{6} \xi$ and $\xi \gg 1 / 6$. Finally, we can write $N$ as a function of $X$ in that limit:

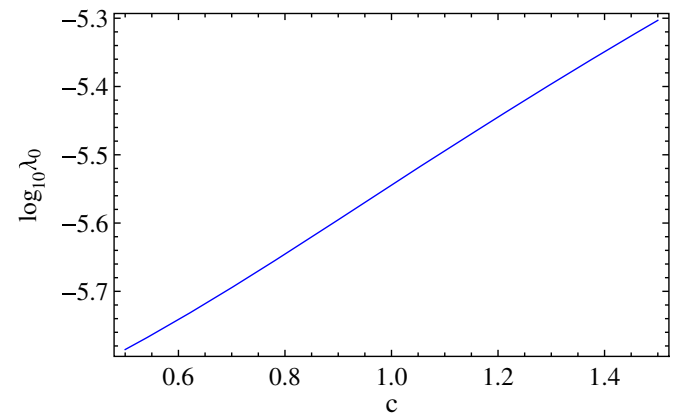

$$
N \simeq \int_{X_{\mathrm{end}}}^{X_{*}} d X \frac{3 X^{3}}{1+X^{2}} \frac{\lambda_{\min }+\frac{\beta_{2}}{\left(16 \pi^{2}\right)^{2}}\left(\ln \frac{X}{c}\right)^{2}}{2 \lambda_{\min }+\frac{\beta_{2}}{\left(16 \pi^{2}\right)^{2}} \ln \frac{X}{c}\left(1+X^{2}+2 \ln \frac{X}{c}\right)} .
$$

This is also in agreement with Ref. [36] if we put $\beta_{2}=0$ and $X_{*} \gg 1$.

\section{Results in prescription II}

Let us numerically estimate the lowest possible value of $\lambda_{\min }$ that allows $U(\varphi)$ to be monotonically increasing. We call this value $\lambda_{0}$. In prescription I, such a value was $\lambda_{c}$, whereas in prescription II, $\lambda_{0}$ is a function of $\beta_{2}$ and $c$. Note that $\lambda_{0}$ is independent of $\xi$ because the expression in the braces in Eq. (57) only depends on $X$, and explicit dependence on $\xi$ drops out of it. In Fig. 9, we plot $\lambda_{0}$ and the position of the saddle point $\varphi_{0}$ as functions of $c$ for a fixed $\beta_{2}=0.5$. We see that $\lambda_{0} \sim 10^{-5.5}$ and $\varphi_{0} \simeq 0.73 \mu_{\text {min }}$ for $c=1$.

The potential is determined by $\lambda_{\min }, c$, and $\xi$. To be specific, we consider the $c=1$ case hereafter. We plot the $\epsilon_{V}$ in Fig. 10 with $c=1, \beta_{2}=0.5$, and $\lambda_{\min }=\lambda_{0}$. The solid and dashed lines represent $\epsilon_{V}$ and $\eta_{V}$, respectively. We can see that $\epsilon_{V} \simeq \eta_{V} \simeq 1$ around $X \simeq 2$. Therefore the end of inflation corresponds to $X_{\text {end }} \simeq 2$.

We can calculate the prediction of inflationary parameters with $c=1, \beta_{2}=0.5$, and $\lambda_{\min }=\lambda_{0} . N=50$ and 65 correspond to $X_{*} \simeq 360$ and 790 , respectively. We fix $\xi$ in such a way that Planck normalization is satisfied,

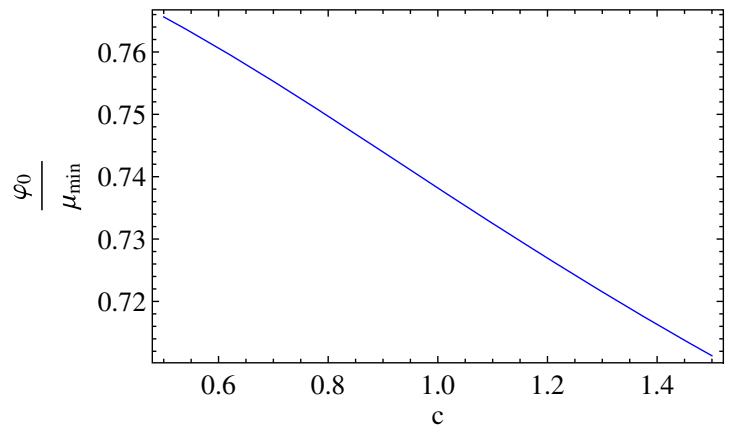

FIG. 9 (color online). Left: $\lambda_{0}$, the minimal value of $\lambda_{\min }$ to maintain monotonicity of the potential, as a function of $c$. Right: $\varphi_{0}$, the position of the saddle point when we set $\lambda_{\min }=\lambda_{0}$, as a function of $c$. 


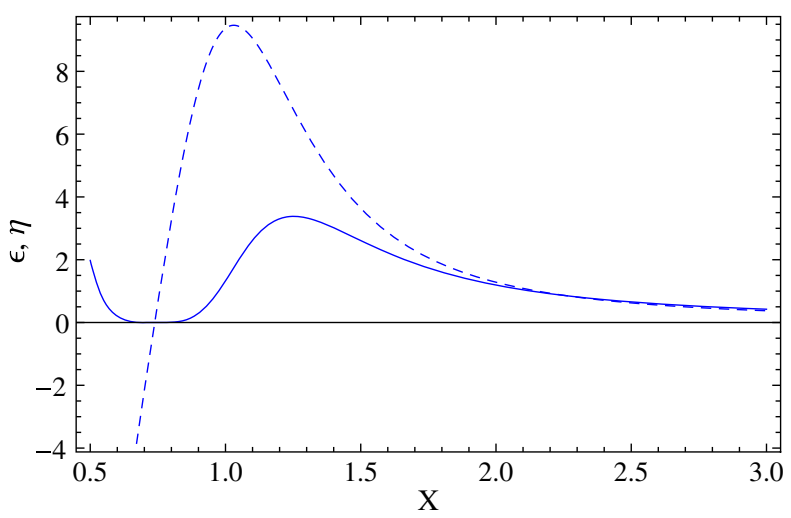

FIG. 10 (color online). Slow-roll parameters $\epsilon$ (solid) and $\eta$ (dashed) as functions of $X=\varphi /\left(M_{P} / \sqrt{\xi}\right)$. We have set $c=1$, $\beta_{2}=0.5$, and $\lambda_{\min }=\lambda_{0}$.

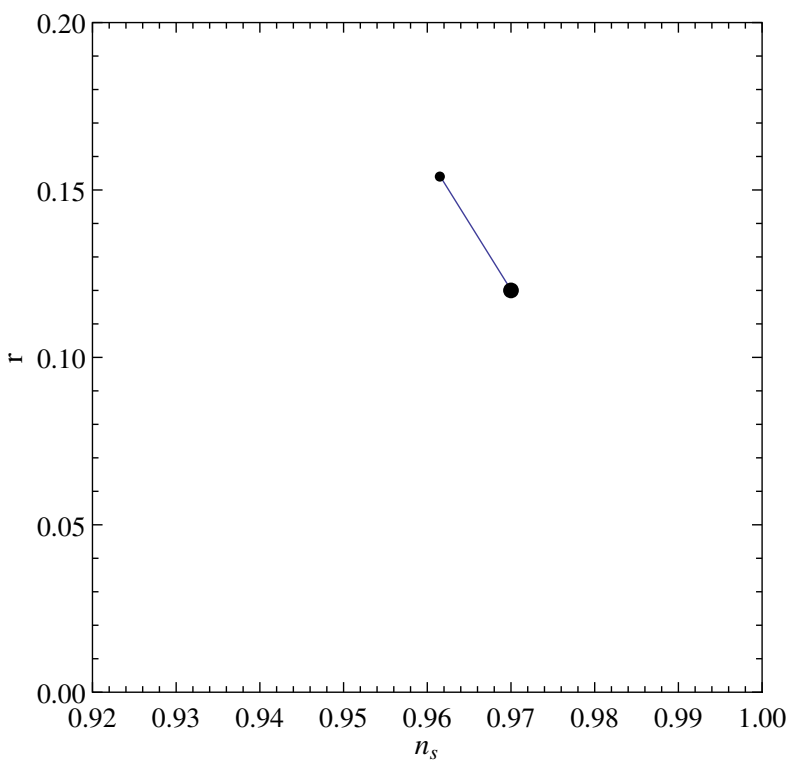

FIG. 11 (color online). $n_{s}$ vs $r$. The small and large dots represent $N_{*}=50$ and 65 .

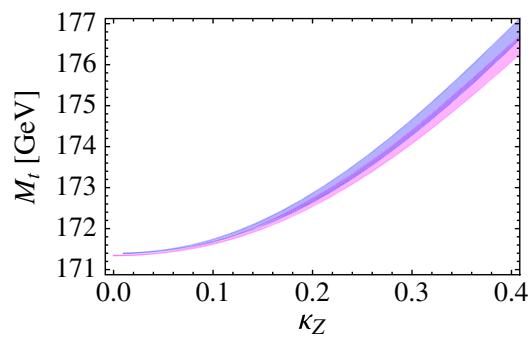

$$
A_{s}=\frac{U}{24 \pi^{2} M_{P}^{4} \epsilon_{V}}=2.2 \times 10^{-9} \text {. }
$$

By using this condition, $\xi$ becomes 190 and 240 for $N=50$ and 65 , respectively. The prediction of $n_{s}$ and $r$ is shown in Fig. 11. $\mathrm{d} n_{s} / \mathrm{d} \ln k$ is small in this case, $\mathrm{d} n_{s} / \mathrm{d} \ln k \ll$ $O\left(10^{-2}\right)$. These predictions are just the same as the chaotic inflation, as discussed in Ref. [48].

We note that the argument in this subsection implicitly assumes that Planck scale physics does not modify the Higgs potential above the UV cutoff.

\section{SCALAR DARK MATTER MODEL}

Next we consider the model which includes Higgs portal singlet scalar DM $S[125,126]$; see also Ref. [127]. The Lagrangian is [128]

$\mathcal{L}=\mathcal{L}_{\mathrm{SM}}+\frac{1}{2}\left(\partial_{\mu} S\right)^{2}-\frac{1}{2} m_{S}^{2} S^{2}-\frac{\rho}{4 !} S^{4}-\frac{\kappa}{2} S^{2} H^{\dagger} H$.

We put subscript $Z$ on the new parameters at the $Z$ mass scale $\mu=M_{Z}$, that is, $\kappa_{Z}=\kappa\left(\mu=M_{Z}\right)$, and $\rho_{Z}=$ $\rho\left(\mu=M_{Z}\right)$. If we require perturbativity up to the cutoff scale, these parameters should be $\kappa_{Z} \lesssim 0.4$ and $\rho_{Z} \lesssim 0.6$ [129]. The one-loop effective potential in this model is given by

$$
\begin{aligned}
& V=V_{\text {tree }}+\Delta V_{1 \text {-loop,DM }} \text {, } \\
& V_{\text {tree }}=e^{4 \Gamma(\varphi)} \frac{\lambda(\mu)}{4} \varphi^{4}, \\
& \Delta V_{1 \text { loop,DM }}=\Delta V_{1 \text {-loop }}+\frac{m_{\mathrm{DM}}^{4}}{64 \pi^{2}}\left(\ln \frac{m_{\mathrm{DM}}(\varphi)^{2}}{\mu^{2}}-\frac{3}{2}\right) \text {, }
\end{aligned}
$$

where $m_{\mathrm{DM}}(\varphi)=\sqrt{\frac{\kappa \varphi^{2}}{2} e^{2 \Gamma(\varphi)}+m_{S}^{2}} \cdot \Delta V_{1-\text { loop }}$ and $\Gamma$ are given by SM one-loop potential 12 and Eq. (5), respectively.

We plot $M_{t}, \beta_{2}$, and $\mu_{\min }$ as functions of $\kappa_{Z}$ imposing the existence of the saddle point in Fig. 12. Here we use two-loop RGEs [129] and put $M_{H}=125.9 \mathrm{GeV}, \alpha_{s}=0.1184$. The band width comes from the requirement of perturbativity of $\rho$ up to string scale [129]: $0 \leq \rho_{Z} \leq 0.6$. The red (lower) and blue (upper) bands correspond to the tree and one-loop
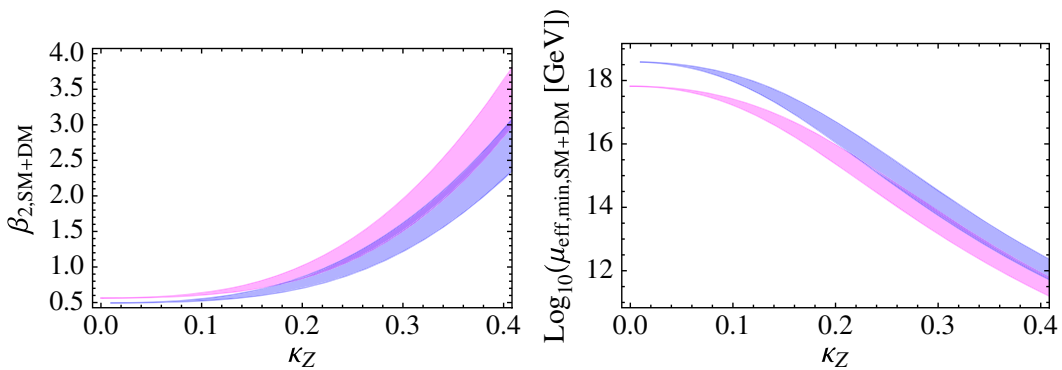

FIG. 12 (color online). $\quad M_{t}$ (left), $\beta_{2}$ (center), and $\mu_{\text {min }}$ (right) are plotted as functions of $\kappa_{Z}$. Red (lower) and blue (upper) bands correspond to the tree and one-loop potentials, respectively. The band width comes from the requirement of perturbativity of $\rho$ up to the string scale [129]: $0 \leq \rho_{Z} \leq 0.6 . M_{H}$ and $m_{S}$ are set to be 125.9 and $0 \mathrm{GeV}$, respectively. 
effective potentials, respectively. From this figure, we see that $\mu_{\min }$ can become smaller than $M_{P}$ by adding $\kappa$ and that $\beta_{2}$ remains to be $O(1)$. In particular, the addition of the scalar DM does not alter the existence of the minimum of $\lambda_{\mathrm{eff}}(\mu)$, which is essential in this inflation scenario with criticality.

\section{SUMMARY}

We have considered the Higgs inflation model which contains nonminimal coupling $\xi \varphi^{2} \mathcal{R}$ [36]. Conventional wisdom was that a large nonminimal coupling $\xi \sim 10^{4}$ is required to fit the cosmic background explorer normalization, $\delta T / T \sim 10^{-5}$, and cosmological predictions are $n_{s}=0.967$ and the small tensor-to-scalar ratio, $r=3 \times 10^{-3}$. In article [48], we have reconsidered this model in light of the discovery of the Higgs boson, which indicates the criticality of the SM. That is, if the SM parameters are tuned so that the saddle point appears, it is possible to realize a Higgs inflation with moderate $\xi$ and generate $O(0.1)$ tensor-to-scalar ratio $r$. The value of $\xi$ is $O(10)$ for prescription I and $O(100)$ for prescription II.

In this paper, we investigate the cosmological predictions of this Higgs inflation in greater detail. To realize this Higgs inflation scenario, it is essential that the effective Higgs quartic coupling $\lambda_{\text {eff }}$ takes its minimum around the scale $10^{17-18} \mathrm{GeV}$. The Higgs potential around the inflation scale is determined by the position $\mu_{\min }$ of the minimum of $\lambda_{\text {eff }}$, the minimum value $\lambda_{\min }$, and the second derivative $\beta_{2}$ around the minimum, in addition to the nonminimal coupling $\xi$. We calculate the cosmological predictions as functions of the above parameters. We also estimate the effects of higher dimensional operators by considering the $\lambda_{6} \varphi^{6} / M_{P}^{2}$ term as an example. We find that the coefficients of the higher dimensional operators should be as small as
$10^{-8}$ in order to account for the scalar and tensor perturbations consistent with the Planck and BICEP2 results. Although we have concentrated on the SM and the Higgs portal scalar DM model in this paper, one may apply our analysis to the other models beyond the SM by evaluating $\lambda_{\min }, \mu_{\min }, \beta_{2}$ in terms of the model parameters.

Finally, we comment on the problem of unitarity [62,130-135]. The problem of unitarity does not threaten the consistency of the Higgs inflation by itself. Concretely speaking, the physical momentum scale during the inflation, which is given by the de Sitter temperature $H_{\text {inf }} \simeq 10^{14} \mathrm{GeV}(r / 0.2)^{1 / 2}$, is smaller than the unitarity violation scale $M_{P} / \xi$ that is evaluated on the electroweak vacuum. ${ }^{11}$ In general, a new physics would appear around the unitarity violation scale. It is interesting that it is around the grand unified theory or string scale in our model.

\section{ACKNOWLEDGMENTS}

The work of Y. H. is supported by the Grant-in-Aid from the Japan Society for the Promotion of Science (JSPS) Grant No. 25.1107. S. C. P. is supported by Basic Science Research Program through the National Research Foundation of Korea funded by the Ministry of Science, ICT \& Future planning (Grants No. NRF-2011-0029758 and No. NRF2013R1A1A2064120). The work of K. O. is in part supported by the Grant-in-Aid for Scientific Research Grants No. 23104009, No. 20244028, and No. 23740192.

\footnotetext{
${ }^{11}$ In Ref. [135], it is shown that the unitarity constraints are relieved for moderate values of nonminimal coupling, $\xi=O(10-100)$
}

[1] J. Beringer et al. (Particle Data Group), 2013 review of particle physics, Phys. Rev. D 86, 010001 (2012).

[2] CMS Collaboration, Precise determination of the mass of the Higgs boson and studies of the compatibility of its couplings with the standard model (2014).

[3] G. Aad et al. (ATLAS Collaboration), Measurement of the Higgs boson mass from the $H \rightarrow \gamma \gamma$ and $H \rightarrow Z Z^{*} \rightarrow 4 \ell$ channels with the ATLAS detector using $25 \mathrm{fb}^{-1}$ of $p p$ collision data Phys. Rev. D 90, 052004 (2014).

[4] M. Holthausen, K. S. Lim, and M. Lindner, Planck scale boundary conditions and the Higgs mass, J. High Energy Phys. 02 (2012) 037.

[5] F. Bezrukov, M. Y. Kalmykov, B. A. Kniehl, and M. Shaposhnikov, Higgs boson mass and new physics, J. High Energy Phys. 10 (2012) 140.

[6] G. Degrassi, S. Di Vita, J. Elias-Miro, J. R. Espinosa, G. F. Giudice, G. Isidori, and A. Strumia, Higgs mass and vacuum stability in the standard model at NNLO, J. High Energy Phys. 08 (2012) 098.

[7] S. Alekhin, A. Djouadi, and S. Moch, The top quark and Higgs boson masses and the stability of the electroweak vacuum, Phys. Lett. B 716, 214 (2012).

[8] I. Masina, The Higgs boson and top quark masses as tests of electroweak vacuum stability, Phys. Rev. D 87, 053001 (2013).

[9] Y. Hamada, H. Kawai, and K.-y. Oda, Bare Higgs mass at Planck scale, Phys. Rev. D 87, 053009 (2013); 89, 059901 (2014).

[10] F. Jegerlehner, The standard model as a low energy effective theory: What is triggering the Higgs mechanism?, Acta Phys. Pol. B 45, 1167 (2014).

[11] D. Buttazzo, G. Degrassi, P. P. Giardino, G. F. Giudice, F. Sala, A. Salvio, and A. Strumia, Investigating the near criticality of the Higgs boson, J. High Energy Phys. 12 (2013) 089. 
[12] V. Branchina and E. Messina, Stability, Higgs Boson Mass, and New Physics, Phys. Rev. Lett. 111, 241801 (2013).

[13] A. Kobakhidze and A. Spencer-Smith, The Higgs vacuum is unstable, arXiv:1404.4709.

[14] A. Spencer-Smith, Higgs vacuum stability in a massdependent renormalization scheme, arXiv:1405.1975.

[15] V. Branchina, E. Messina, and A. Platania, Top mass determination, Higgs inflation, and vacuum stability, J. High Energy Phys. 09 (2014) 182.

[16] V. Branchina, E. Messina, and M. Sher, The lifetime of the electroweak vacuum and sensitivity to Planck scale physics, Phys. Rev. D 91, 013003 (2015).

[17] Y. Hamada, H. Kawai, and K.-y. Oda, Bare Higgs mass and potential at ultraviolet cutoff, in Proceedings for Toyama International Workshop on Higgs as a Probe of New Physics, 2013 edited by S. Kanemura and T. Shindou, SLAC eConf C130213.1 (2013)

[18] I. Masina and M. Quiros, On the Veltman condition, the hierarchy problem, and high-scale supersymmetry, Phys. Rev. D 88, 093003 (2013).

[19] M. T. Frandsen, F. Sannino, I. M. Shoemaker, and O. Svendsen, LUX constraints on magnetic dark matter in the $S \bar{E} \chi$ y model with(out) naturality, Phys. Rev. D 89, 055004 (2014).

[20] M. Al-sarhi, I. Jack, and D. Jones, Quadratic divergences in gauge theories, Z. Phys. C 55, 283 (1992).

[21] D. Jones, The quadratic divergence in the Higgs mass revisited, Phys. Rev. D 88, 098301 (2013).

[22] N. Haba, K. Kaneta, and R. Takahashi, Planck scale boundary conditions in the standard model with singlet scalar dark matter, J. High Energy Phys. 04 (2014) 029.

[23] O. Antipin, M. Mojaza, and F. Sannino, Conformal extensions of the standard model with Veltman conditions, Phys. Rev. D 89, 085015 (2014).

[24] O. Antipin, J. Krog, M. Mojaza, and F. Sannino, A stable extension with(out) gravity, Nucl. Phys. B886, 125 (2014).

[25] L. Bian, RGE, the naturalness problem, and the understanding of the Higgs mass term, Phys. Rev. D 88, 056022 (2013).

[26] S. Iso, N. Okada, and Y. Orikasa, Classically conformal B$L$ extended standard model, Phys. Lett. B 676, 81 (2009).

[27] S. Iso, N. Okada, and Y. Orikasa, The minimal $B-L$ model naturally realized at TeV scale, Phys. Rev. D 80, 115007 (2009).

[28] H. Aoki and S. Iso, Revisiting the naturalness problemWho is afraid of quadratic divergences?, Phys. Rev. D 86, 013001 (2012).

[29] S. Iso and Y. Orikasa, TeV Scale B-L model with a flat Higgs potential at the Planck scale-in view of the hierarchy problem, Prog. Theor. Exp. Phys. 2013, 023B08 (2013).

[30] M. Hashimoto, S. Iso, and Y. Orikasa, Radiative symmetry breaking at the Fermi scale and flat potential at the Planck scale, Phys. Rev. D 89, 016019 (2014).

[31] M. Hashimoto, S. Iso, and Y. Orikasa, Radiative symmetry breaking from flat potential in various U(1)' models, Phys. Rev. D 89, 056010 (2014).

[32] P. H. Chankowski, A. Lewandowski, K. A. Meissner, and H. Nicolai, Softly broken conformal symmetry and the stability of the electroweak scale, Mod. Phys. Lett. A 30, 1550006 (2015).

[33] A. Kobakhidze and K. L. McDonald, Comments on the hierarchy problem in effective theories, J. High Energy Phys. 07 (2014) 155.

[34] Y. Hamada, H. Kawai, and K.-y. Oda, Minimal higgs inflation, Prog. Theor. Exp. Phys. 2014, 23 B02 (2014).

[35] C. Froggatt and H. B. Nielsen, Standard model criticality prediction: Top mass $173+-5-\mathrm{GeV}$ and Higgs mass $135+-9-G e V$, Phys. Lett. B 368, 96 (1996).

[36] F. L. Bezrukov and M. Shaposhnikov, The standard model Higgs boson as the inflaton, Phys. Lett. B 659, 703 (2008).

[37] I. Masina and A. Notari, The Higgs mass range from standard model false vacuum Inflation in scalar-tensor gravity, Phys. Rev. D 85, 123506 (2012).

[38] I. Masina and A. Notari, Standard Model False Vacuum Inflation: Correlating the Tensor-to-Scalar Ratio to the Top Quark and Higgs Boson Masses, Phys. Rev. Lett. 108, 191302 (2012).

[39] I. Masina and A. Notari, Inflation from the Higgs field false vacuum with hybrid potential, J. Cosmol. Astropart. Phys. 11 (2012) 031.

[40] M. Fairbairn, P. Grothaus, and R. Hogan, The problem with false vacuum Higgs inflation, J. Cosmol. Astropart. Phys. 06 (2014) 039.

[41] A. Barvinsky, A. Y. Kamenshchik, and A. Starobinsky, Inflation scenario via the standard model Higgs boson and LHC, J. Cosmol. Astropart. Phys. 11 (2008) 021.

[42] A. De Simone, M. P. Hertzberg, and F. Wilczek, Running inflation in the standard model, Phys. Lett. B 678, 1 (2009).

[43] F. L. Bezrukov, A. Magnin, and M. Shaposhnikov, Standard model Higgs boson mass from inflation, Phys. Lett. B 675, 88 (2009).

[44] F. Bezrukov and M. Shaposhnikov, Standard model Higgs boson mass from inflation: Two-loop analysis, J. High Energy Phys. 07 (2009) 089.

[45] F. Bezrukov, A. Magnin, M. Shaposhnikov, and S. Sibiryakov, Higgs inflation: Consistency and generalizations, J. High Energy Phys. 01 (2011) 016.

[46] A. Salvio, Higgs inflation at NNLO after the boson discovery, Phys. Lett. B 727, 234 (2013).

[47] Allison Kyle, Higgs xi-inflation for the $125-126 \mathrm{GeV}$ Higgs: A two-loop analysis, J. High Energy Phys. 02 (2014) 040.

[48] Y. Hamada, H. Kawai, K.-y. Oda, and S. C. Park, Higgs Inflation Still Alive, Phys. Rev. Lett. 112, 241301 (2014).

[49] J. L. Cook, L. M. Krauss, A. J. Long, and S. Sabharwal, Is Higgs inflation dead?, Phys. Rev. D 89, 103525 (2014).

[50] F. Bezrukov and M. Shaposhnikov, Higgs inflation at the critical point, Phys. Lett. B 734, 249 (2014).

[51] S. Kawai and N. Okada, TeV scale seesaw from supersymmetric Higgs-lepton inflation and BICEP2, Phys. Lett. B 735, 186 (2014).

[52] N. Haba and R. Takahashi, Higgs inflation with singlet scalar dark matter and right-hand neutrino in the light of BICEP2, Phys. Rev. D 89, 115009 (2014). 
[53] P. Ko and W.-I. Park, Higgs-portal assisted Higgs inflation in light of BICEP2, arXiv:1405.1635.

[54] N. Haba, H. Ishida, and R. Takahashi, Higgs inflation and Higgs portal dark matter with right-hand neutrinos, arXiv:1405.5738.

[55] H.-J. He and Z.-Z. Xianyu, Extending Higgs inflation with $\mathrm{TeV}$ scale new physics, J. Cosmol. Astropart. Phys. 10 (2014) 019.

[56] C. Germani and A. Kehagias, New Model of Inflation with Nonminimal Derivative Coupling of Standard Model Higgs Boson to Gravity, Phys. Rev. Lett. 105, 011302 (2010).

[57] K. Kamada, T. Kobayashi, M. Yamaguchi, and J. Yokoyama, Higgs G-inflation, Phys. Rev. D 83, 083515 (2011).

[58] K. Kamada, T. Kobayashi, T. Takahashi, M. Yamaguchi, and J. Yokoyama, Generalized Higgs inflation, Phys. Rev. D 86, 023504 (2012).

[59] G. Chakravarty, S. Mohanty, and N. K. Singh, Higgs inflation in $f(\Phi, R)$ theory, Int. J. Mod. Phys. D D23, 1450029 (2014).

[60] K. Kamada, T. Kobayashi, T. Kunimitsu, M. Yamaguchi, and J. Yokoyama, Graceful exit from Higgs G-inflation, Phys. Rev. D 88, 123518 (2013).

[61] K. Nakayama and F. Takahashi, Higgs chaotic inflation and the primordial b-mode polarization discovered by BICEP2, Phys. Lett. B 734, 96 (2014).

[62] H. M. Lee, Chaotic inflation and unitarity problem, Eur. Phys. J. C 74, 3022 (2014).

[63] J. Rubio and M. Shaposhnikov, Higgs-dilaton cosmology: Universality vs criticality, Phys. Rev. D 90, 027307 (2014).

[64] Y. Hosotani, Stability of scalar fields in curved space, Phys. Rev. D 32, 1949 (1985).

[65] I. Ben-Dayan and R. Brustein, Cosmic microwave background observables of small field models of inflation, J. Cosmol. Astropart. Phys. 09 (2010) 007.

[66] T. Kubota, N. Misumi, W. Naylor, and N. Okuda, The conformal transformation in general single field inflation with nonminimal coupling, J. Cosmol. Astropart. Phys. 02 (2012) 034.

[67] I. Ben-Dayan and T. Kalaydzhyan, Constraining the primordial power spectrum from SNIa lensing dispersion, Phys. Rev. D 90, 083509 (2014).

[68] F. Jegerlehner, Higgs inflation and the cosmological constant, Acta Phys. Pol. B 45, 1215 (2014).

[69] D. Lindley, Focus: Theorists weigh in on BICEP2, Physics 7, 65 (2014).

[70] C. Cheng, Q. Huang, and W. Zhao, Constraints on the extensions to the base $\Lambda$ CDM model from BICEP2, Planck, and WMAP, Sci. China Phys. Mech. Astron. 57, 1460 (2014).

[71] C. Burgess, S. P. Patil, and M. Trott, On the predictiveness of single-field inflationary models, J. High Energy Phys. 06 (2014) 010.

[72] Q. Gao and Y. Gong, The challenge for single-field inflation with BICEP2 result, Phys. Lett. B 734, 41 (2014).

[73] N. Okada, V. N. Senoguz, and Q. Shafi, Simple inflationary models in light of BICEP2: an update, arXiv:1403.6403.
[74] I. Oda and T. Tomoyose, Quadratic chaotic inflation from Higgs inflation, Adv. Stud. Theor. Phys. 8, 551 (2014).

[75] K. Enqvist, S. Nurmi, and S. Rusak, Non-Abelian dynamics in the resonant decay of the Higgs after inflation, J. Cosmol. Astropart. Phys. 10 (2014) 064.

[76] K. Enqvist, T. Meriniemi, and S. Nurmi, Higgs dynamics during Inflation, J. Cosmol. Astropart. Phys. 07 (2014) 025 .

[77] K. Bamba, G. Cognola, S. D. Odintsov, and S. Zerbini, One-loop modified gravity in de Sitter universe, quantum corrected inflation, and its confrontation with the Planck result, Phys. Rev. D 90, 023525 (2014).

[78] P. Channuie and K. Karwan, Large tensor-to-scalar ratio from composite inflation, Phys. Rev. D 90, 047303 (2014).

[79] T. Higaki, R. Kitano, and R. Sato, Neutrinoful universe, J. High Energy Phys. 07 (2014) 044.

[80] K. Kannike, A. Racioppi, and M. Raidal, Embedding inflation into the standard model-more evidence for classical scale invariance, J. High Energy Phys. 06 (2014) 154.

[81] Q. Gao, Y. Gong, and T. Li, The modified Lyth bound and implications of BICEP2 results, Phys. Rev. D 91, 063509 (2015).

[82] N. Haba, H. Ishida, K. Kaneta, and R. Takahashi, Vanishing Higgs potential at the Planck scale in singlets extension of the standard model, Phys. Rev. D 90, 036006 (2014).

[83] M. Rinaldi, G. Cognola, L. Vanzo, and S. Zerbini, Reconstructing the inflationary $f(R)$ from observations, J. Cosmol. Astropart. Phys. 08 (2014) 015.

[84] B. Chen and Z.-w. Jin, Anisotropy in inflation with nonminimal coupling, J. Cosmol. Astropart. Phys. 09 (2014) 046.

[85] K. Bamba, S. Nojiri, and S. D. Odintsov, Reconstruction of scalar field theories realizing inflation consistent with the Planck and BICEP2 results, Phys. Lett. B 737, 374 (2014).

[86] M. Postma and M. Volponi, Equivalence of the Einstein and Jordan frames, Phys. Rev. D 90, 103516 (2014).

[87] Z.-Z. Xianyu and H.-J. He, Asymptotically safe Higgs inflation, J. Cosmol. Astropart. Phys. 10 (2014) 083.

[88] T. Li, Z. Sun, C. Tian, and L. Wu, The renormalizable three-term polynomial inflation with large tensor-to-scalar ratio, arXiv:1407.8063.

[89] T. Inagaki, R. Nakanishi, and S. D. Odintsov, Inflationary parameters in renormalization group improved $\phi^{4}$ theory, Astrophys. Space Sci. 354, 2108 (2014).

[90] E. Elizalde, S. Odintsov, E. Pozdeeva, and S. Y. Vernov, Renormalization-group inflationary scalar electrodynamics and SU(5) scenarios confronted with Planck2013 and BICEP2 results, Phys. Rev. D 90, 084001 (2014).

[91] I. Ben-Dayan, in Proceedings of the XLIXth Rencontres de Moriond, Cosmology Session (La Thuile, 2014).

[92] K. Bhattacharya, J. Chakrabortty, S. Das, and T. Mondal, Higgs vacuum stability and inflationary dynamics in the light of BICEP2 results, J. Cosmol. Astropart. Phys. 12 (2014) 001.

[93] S. Kawabata, Y. Shimizu, Y. Sumino, and H. Yokoya, Weight function method for precise determination of top quark mass at Large Hadron Collider, Phys. Lett. B 741, 232 (2015). 
[94] S. Kawabata, in TOP LHC WG meeting, 2014, CERN, https://indico.cern.ch/event/301787/other-view.

[95] L. Di Luzio and L. Mihaila, On the gauge dependence of the standard model vacuum instability scale, J. High Energy Phys. 06 (2014) 079.

[96] N. Nielsen, Removing the gauge parameter dependence of the effective potential by a field redefinition, Phys. Rev. D 90, 036008 (2014).

[97] A. Andreassen, W. Frost, and M. D. Schwartz, Consistent use of effective potentials, Phys. Rev. D 91, 016009 (2015).

[98] A. Andreassen, W. Frost, and M. D. Schwartz, Consistent Use of the Standard Model Effective Potential, Phys. Rev. Lett. 113, 241801 (2014).

[99] S. Moch, S. Weinzierl, S. Alekhin, J. Blumlein, L. de la Cruz et al., High precision fundamental constants at the TeV scale, arXiv:1405.4781.

[100] I. L. Shapiro and H. Takata, Conformal transformation in gravity, Phys. Lett. B 361, 31 (1995).

[101] S. C. Park and S. Yamaguchi, Inflation by nonminimal coupling, J. Cosmol. Astropart. Phys. 08 (2008) 009.

[102] P. Ade et al. (Planck Collaboration), Planck 2013 results. XXII. Constraints on inflation, Astron. Astrophys. 571, A22 (2014).

[103] P. Ade et al. (BICEP2 Collaboration), Detection of BMode Polarization at Degree Angular Scales by BICEP2, Phys. Rev. Lett. 112, 241101 (2014).

[104] M. J. Mortonson and U. Seljak, A joint analysis of Planck and BICEP2 B modes including dust polarization uncertainty, J. Cosmol. Astropart. Phys. 10 (2014) 035.

[105] R. Flauger, J. C. Hill, and D. N. Spergel, Toward an understanding of foreground emission in the BICEP2 region, J. Cosmol. Astropart. Phys. 08 (2014) 039.

[106] M. Kawasaki, T. Sekiguchi, T. Takahashi, and S. Yokoyama, Isocurvature perturbations and tensor mode in light of Planck and BICEP2, J. Cosmol. Astropart. Phys. 08 (2014) 043.

[107] J.-F. Zhang, Y.-H. Li, and X. Zhang, Sterile neutrinos help reconcile the observational results of primordial gravitational waves from Planck and BICEP2, Phys. Lett. B 740, 359 (2015).

[108] C. Froggatt, H. B. Nielsen, and Y. Takanishi, Standard model Higgs boson mass from borderline metastability of the vacuum, Phys. Rev. D 64, 113014 (2001).

[109] H. B. Nielsen, in Proceedings to the 15th Bled Workshop "What Comes Beyond Standard Models," DMFA ZALOZNISTVO, 2012 (Bled workshops in physics, Ljubljana, 2012), p. 94.

[110] K. A. Meissner and H. Nicolai, Conformal symmetry and the standard model, Phys. Lett. B 648, 312 (2007).

[111] R. Foot, A. Kobakhidze, K. L. McDonald, and R. R. Volkas, A solution to the hierarchy problem from an almost decoupled hidden sector within a classically scale invariant theory, Phys. Rev. D 77, 035006 (2008).

[112] K. A. Meissner and H. Nicolai, Effective action, conformal anomaly, and the issue of quadratic divergences, Phys. Lett. B 660, 260 (2008).

[113] M. Shaposhnikov and C. Wetterich, Asymptotic safety of gravity and the Higgs boson mass, Phys. Lett. B 683, 196 (2010).
[114] Y. Kawamura, Naturalness, conformal symmetry, and duality, Prog. Theor. Exp. Phys. 2013 (2013) 113 B04.

[115] Y. Kawamura, Gauge hierarchy problem, supersymmetry, and fermionic symmetry, arXiv:1311.2365.

[116] H. Kawai and T. Okada, Solving the naturalness problem by baby universes in the Lorentzian multiverse, Prog. Theor. Phys. 127, 689 (2012).

[117] H. Kawai, Low energy effective action of quantum gravity and the naturalness problem, Int. J. Mod. Phys. A A28, 1340001 (2013).

[118] Y. Hamada, H. Kawai, and K. Kawana, Evidence of the big fix, Int. J. Mod. Phys. A A29, 1450099 (2014).

[119] K. Kawana, Reconsideration of the Coleman's baby universe, arXiv:1405.2743.

[120] I. Buchbinder, S. Odintsov, and I. Shapiro, Effective action in quantum gravity (Bristol, UK, 1992), p. 413.

[121] S. D. Odintsov, Renormalization group, effective action, and grand unification theories in curved spacetime, Fortschr. Phys. 39, 621 (1991).

[122] T. Muta and S. Odintsov, Model dependence of the nonminimal scalar graviton effective coupling constant in curved spacetime, Mod. Phys. Lett. A 06, 3641 (1991).

[123] Y. Yoon and Y. Yoon, Asymptotic conformal invariance of SU(2) and standard models in curved spacetime, Int. J. Mod. Phys. A A12, 2903 (1997).

[124] F. Bezrukov, The Higgs field as an inflaton, Classical Quantum Gravity 30, 214001 (2013).

[125] C. Burgess, M. Pospelov, and T. ter Veldhuis, The minimal model of nonbaryonic dark matter: A singlet scalar, Nucl. Phys. B619, 709 (2001).

[126] J. M. Cline, K. Kainulainen, P. Scott, and C. Weniger, Update on scalar singlet dark matter, Phys. Rev. D 88, 055025 (2013).

[127] C. Kim, S. C. Park, K. Wang, and G. Zhu, Invisible Higgs decay with $B \rightarrow K \nu \bar{\nu}$ constraint, Phys. Rev. D 81, 054004 (2010).

[128] V. Silveira and A. Zee, Scalar phantoms, Phys. Lett. 161B, 136 (1985).

[129] Y. Hamada, H. Kawai, and K.-y. Oda, Predictions on mass of Higgs portal scalar dark matter from Higgs inflation and flat potential, J. High Energy Phys. 07 (2014) 026.

[130] C. P. Burgess, Hyun Min Lee, and Michael Trott, Powercounting and the validity of the classical approximation during inflation, J. High Energy Phys. 09 (2009) 103.

[131] J. L. F. Barbon and J. R. Espinosa, On the naturalness of Higgs inflation, Phys. Rev. D 79, 081302 (2009).

[132] C. P. Burgess, Hyun Min Lee, and Michael Trott, Comment on Higgs inflation and naturalness, J. High Energy Phys. 07 (2010) 007.

[133] M. P. Hertzberg, On inflation with nonminimal coupling, J. High Energy Phys. 11 (2010) 023.

[134] Gian F. Giudice and Hyun Min Lee, Unitarizing Higgs inflation, Phys. Lett. B 694, 294 (2011).

[135] J. Ren, Z.-Z. Xianyu, and H.-J. He, Higgs gravitational interaction, weak boson scattering, and Higgs inflation in Jordan and Einstein frames, J. Cosmol. Astropart. Phys. 06 (2014) 032.

[136] K. Olive et al. (Particle Data Group), Review of particle physics, Chin. Phys. C 38, 090001 (2014). 
[137] V. e. Abazov (D0 Collaboration), Precision Measurement of the Top Quark Mass in lepton + jets Final States, Phys. Rev. Lett. 113, 032002 (2014).

[138] Tevatron Electroweak Working Group, Combination of CDF and D0 results on the mass of the top quark using up to $9.7 \mathrm{fb}^{-1}$ at the Tevatron, arXiv:1407.2682.
[139] D0 Collaborations, T. ATLAS, CDF, and CMS, First combination of Tevatron and LHC measurements of the top quark mass, arXiv:1403.4427.

[140] CMS Collaboration, Combination of the CMS top quark mass measurements from run 1 of the LHC (2014). 